Boletín de la Sociedad Geológica Mexicana

VOLUMEN 63, NÚM. 2, 2011, P. 217-233

\title{
Una aproximación a la regionalización sísmica del estado de Nuevo León, basada en velocidades de propagación de ondas de corte y geología
}

\author{
Juan Carlos Montalvo Arrieta ${ }^{1, *}$, Luis Gerardo Ramos Zúñiga ${ }^{1}$, Ignacio Navarro de León ${ }^{1}$, \\ Juan Alonso Ramírez Fernández ${ }^{1}$ \\ ${ }^{1}$ Facultad de Ciencias de la Tierra, Universidad Autónoma de Nuevo León, Carretera a Cerro Prieto km 8, Ex-Hacienda de \\ Guadalupe, 67000 Linares, Nuevo León. \\ *montalvo@fct.uanl.mx
}

\begin{abstract}
Resumen
En este artículo se presenta el primer mapa de la regionalización sísmica del Estado de Nuevo León, escala 1:250000. Éste fue elaborado usando información geológica y datos de velocidad de propagación de ondas P y S. Para la obtención de las velocidades de propagación de ondas se utilizó la metodología de refracción sísmica. Se realizaron un total de 708 perfiles sobre diferentes unidades geológicas que afloran y conforman la columna litológica tipo del Estado de Nuevo León. Los afloramientos en donde se realizaron los perfiles sísmicos comprenden rocas sedimentarias, ígneas, metamórficas y depósitos del Cuaternario. La generación del mapa de las condiciones sísmicas de sitio está basada en códigos de efectos de sitio, como propone el National Earthquake Hazards Reduction Program (NEHRP, EE.UU.), a partir de la determinación de $\mathrm{V}_{\mathrm{S} 30} \mathrm{y}$ su correlación con información litológica. El mapa de regionalización por $\mathrm{V}_{\mathrm{S} 30}$, reveló los sitios clase $\mathrm{A}\left(\mathrm{V}_{\mathrm{S} 30}>1500 \mathrm{~m} / \mathrm{s}\right)$, clase $\mathrm{B}\left(760<\mathrm{V}_{\mathrm{S} 30} \leq 1500\right)$ y clase $\mathrm{C}\left(360<\mathrm{V}_{\mathrm{S} 30} \leq 760\right)$ predominan en el área de estudio. En las regiones caracterizadas por material aluvial muestran valores más bajos de $\mathrm{V}_{\mathrm{S} 30}(560 \mathrm{~m} / \mathrm{s})$, mientras que las rocas más consolidadas como, por ejemplo, las que conforman la secuencia mesozoica, presentan valores altos de propagación de ondas sísmicas $\left(\mathrm{V}_{\mathrm{S} 30}=1200-3500 \mathrm{~m} / \mathrm{s}\right)$. Los resultados generados en este trabajo proveen de una primera aproximación de la respuesta sísmica de los materiales someros en el estado, cuya aplicación es de importancia en la planeación del uso de suelo, desarrollo urbano, así como para el cálculo del riesgo sísmico u otros peligros geológicos, particularmente para regiones donde existe evidencia histórica de actividad sísmica, pero con escasa o nula instrumentación sísmica.
\end{abstract}

Palabras clave: Perfiles sísmicos de refracción, $\mathrm{V}_{\mathrm{S} 30}$, Noreste de México, efectos sísmicos de sitio, sismicidad intraplaca.

\begin{abstract}
This paper presents the first seismic regionalization map for Nuevo Leon state, at a scale of 1:250 000. It was made using geological information and $P$ and $S$ wave propagation velocity data. Seismic refraction methodology was used to obtain the wave velocity propagation. A total of 708 profiles were made on different geologic units that are included in the Nuevo Leon state type-lithological column. Outcrops on which the profiles were carried out include sedimentary, igneous, and metamorphic rocks, and Quaternary deposits. The generation of the seismic site conditions map is based on site effect codes, as proposed by the National Earthquake Hazards Reduction Program (NEHRP, U.S.A.), from $V_{S 30}$ determination and its correlation with lithological information. The regionalization map using $V_{S 30}$, has revealed that $A\left(V_{S 30}>1500 \mathrm{~m} / \mathrm{s}\right), B\left(760<V_{S 30} \leq 1500\right)$, and $C\left(360<V_{S 30} \leq 760\right)$ class sites predominate in the study area. Areas characterized by alluvial material show lower values of $V_{S 30}(560 \mathrm{~m} / \mathrm{sec})$, whereas consolidated rocks, for example, those that constitute the Mesozoic sequences, display high seismic wave velocity propagation values $\left(V_{S 30}=1200-3500 \mathrm{~m} / \mathrm{s}\right)$. The results obtained in this work provide a first approximation of the seismic response of shallow materials in the state, whose use is important
\end{abstract}


in the land use planning, urban development and for the calculation of seismic risk and other geological perils, particularly for areas with historical evidence of seismic activity, but with little or no seismic instrumentation.

Keywords: Seismic refraction profiles, $V_{S 30^{\prime}}$ Northeast Mexico, seismic site effects, intraplate seismicity.

\section{Introducción}

Se ha documentado ampliamente que las condiciones geológicas pueden generar amplificaciones importantes del movimiento del suelo y concentrar el daño en estructuras durante terremotos. De manera general, los perjuicios causados por la sacudida del terremoto comúnmente se relacionan con variaciones de los materiales geológicos cercanos a la superficie y, de manera particular, estas grandes amplificaciones del movimiento del terreno se asocian con depósitos sedimentarios recientes y poco consolidados (Tinsley y Fumal, 1985). Históricamente, los principales núcleos de población en todos los países del mundo se han asentado y desarrollado sobre depósitos aluviales del Cuaternario los cuales, de manera general, están expuestos a diferentes tipos de riesgos geológicos (hundimientos/ colapsos, inundaciones, grandes sacudidas del terreno, entre otros). Debido a esto, es importante la aplicación de técnicas cartográficas especializadas para identificar y evaluar los riesgos geológicos a los cuales están sujetos estos depósitos aluviales.

En regiones propensas a experimentar sacudidas del terreno, es necesario establecer regionalizaciones $\mathrm{y} / \mathrm{o}$ microzonificaciones sísmicas, con la finalidad de subdividir áreas con diferente potencial de peligrosidad ante la incidencia de un terremoto, a partir de la definición de sus comportamientos sísmicos característicos. Esta información es de utilidad para el diseño de estructuras, planeación del uso del suelo, códigos sísmicos, así como para estudios de mitigación del riesgo por terremoto.

Los procesos que controlan el efecto sísmico de sitio y que están directamente relacionados con la distribución de daños son: la litología, los espesores y las propiedades elásticas de los suelos y rocas, la profundidad al macizo rocoso, la profundidad del nivel freático, la topografía y la geometría de cuencas o valles aluviales, así como los procesos de directividad y de anisotropía sísmica (Roca et al., 2008). En particular, en áreas de baja sismicidad y ausencia de registros de movimientos fuertes del terreno, una manera de clasificar, evaluar y cartografiar las condiciones sísmicas de sitio es a través de correlaciones entre geología superficial, datos de pozos (litología, espesores, velocidades de propagación de ondas de corte, resistencia a la penetración o valor N) y mediciones de velocidades superficiales de la propagación de ondas de corte. Estas técnicas han sido utilizadas en diferentes zonas urbanas localizadas muy cercanas a límites de placas, como también en el interior de los continentes (Tinsley y Fumal,
1985; Park y Elrick, 1998; Wills y Silva, 1998; Wills et al., 2000; Rodríguez-Marek et al., 2001; Stewart et al., 2003; Wills y Clan, 2006; Hanumantharo y Ramana, 2008; Montalvo-Arrieta et al., 2008).

Aunque la ocurrencia de grandes terremotos intraplaca es relativamente infrecuente, comparada con los sismos asociados a límites de placas, estos eventos ocasionales pueden resultar extremadamente devastadores. Esto se debe a que la mayoría de las ciudades localizadas en el interior de los continentes han sido edificadas sin tomar en cuenta criterios de diseño sísmico. Se ha definido que los grandes sismos al interior de los continentes pueden causar daños en áreas muy extensas, debido a que la atenuación de su energía sísmica es relativamente baja hacia el interior de las placas (Crone et al., 2003). Stein (2007) mencionó que la sacudida del terreno provocada por sismos intraplaca (por ejemplo, zona sísmica de Nuevo Madrid, centro de EE.UU.), es comparable con aquélla de los sismos que se originan en límites de placa (California) con una unidad de magnitud más grande, ya que las rocas en el interior estable de los continentes transmiten la energía más eficientemente. Un ejemplo de este tipo en la República Mexicana fue el terremoto de Bavispe, Sonora, de 1887 ( $\mathrm{M}_{\mathrm{w}}$ 7.4; Natali y Sbar, 1982). De acuerdo con Aguilera (1888), la distribución de isosistas comprendió desde Santa Fe, Nuevo México, EE.UU. (Intensidad I en la escala de Mercalli Modificada, IMM) en el norte, hasta la Ciudad de México (IMM = I) en el centro del país. En el área epicentral (noroeste de Bavispe, Sonora) se reportaron intensidades de X (IMM). En cambio, para el terremoto de Michoacán de septiembre de $1985\left(M_{w}=8.1\right.$, Servicio Sismológico Nacional, SSN), en el área epicentral las intensidades sísmicas fueron de IX; hacia el norte del país, se reportaron intensidades de II en los límites de Nuevo León y Tamaulipas (México) con el estado de Texas (EE.UU.). A partir de estas observaciones, es necesario conocer leyes de atenuación, así como la respuesta sísmica de sitio para estas regiones intraplaca, con la finalidad de identificar regiones o áreas potenciales de fallar o experimentar importantes amplificaciones del movimiento de suelo ante la sacudida de un terremoto.

El objetivo de este estudio ha sido generar el primer mapa escala 1:250000 de la distribución de las condiciones sísmicas de sitio para el estado de Nuevo León, a partir de velocidades sísmicas de ondas de corte y geología superficial. La clasificación sísmica de sitio se ha realizado de acuerdo con la clasificación general propuesta por la National Earthquake Hazards Reduction Program (NEHRP, EE.UU.). 


\section{Marco sismotectónico}

El estado de Nuevo León se localiza en el noreste de México, en el límite de dos provincias geológicas: la Sierra Madre Oriental (SMO) y la Planicie Costera del Golfo (PCG). Esta región se caracteriza por una sismicidad baja y ausencia de registros de movimientos fuertes. GalvánRamírez y Montalvo-Arrieta (2008) presentaron un catálogo de 148 terremotos, para el periodo 1787 - 2006 (Figura 1). Estos autores mencionaron que los principales sismos en el Norte de México y la región fronteriza México-EE.UU. incluyen: Bavispe, Sonora, en $1887\left(\mathrm{M}_{\mathrm{W}}=7.4\right.$, Natali y Sbar, 1982); Parral, Chihuahua, en $1928\left(\mathrm{M}_{\mathrm{w}}=6.5\right.$, Doser y Rodríguez, 1993); Valentine, Texas, en $1931\left(\mathrm{M}_{\mathrm{w}}=6.4\right.$, Doser, 1987), y Alpine, Texas, en $1995\left(\mathrm{M}_{\mathrm{w}}=5.7\right.$; Xie, 1998; Frohlich y Davis, 2002). El resto de la sismicidad reportada en la región por Galván-Ramírez y MontalvoArrieta (2008) varía $2.3<\mathrm{M}<4.8$.

Para el estado de Nuevo León, históricamente, los sismos que se pueden considerar como los más severos en relación a los daños generados ocurrieron los días 7 y 8 de enero de 1880. García Acosta y Suárez Reynoso (1996) mencionan que en la región de Dr. Arroyo y Mier y Noriega, Nuevo León, se reportaron daños en algunas construcciones así como en la iglesia del primer municipio. Este terremoto también se sintió en Matehuala, San Luis Potosí, y durante cuatro días después se siguieron reportando sismos. De

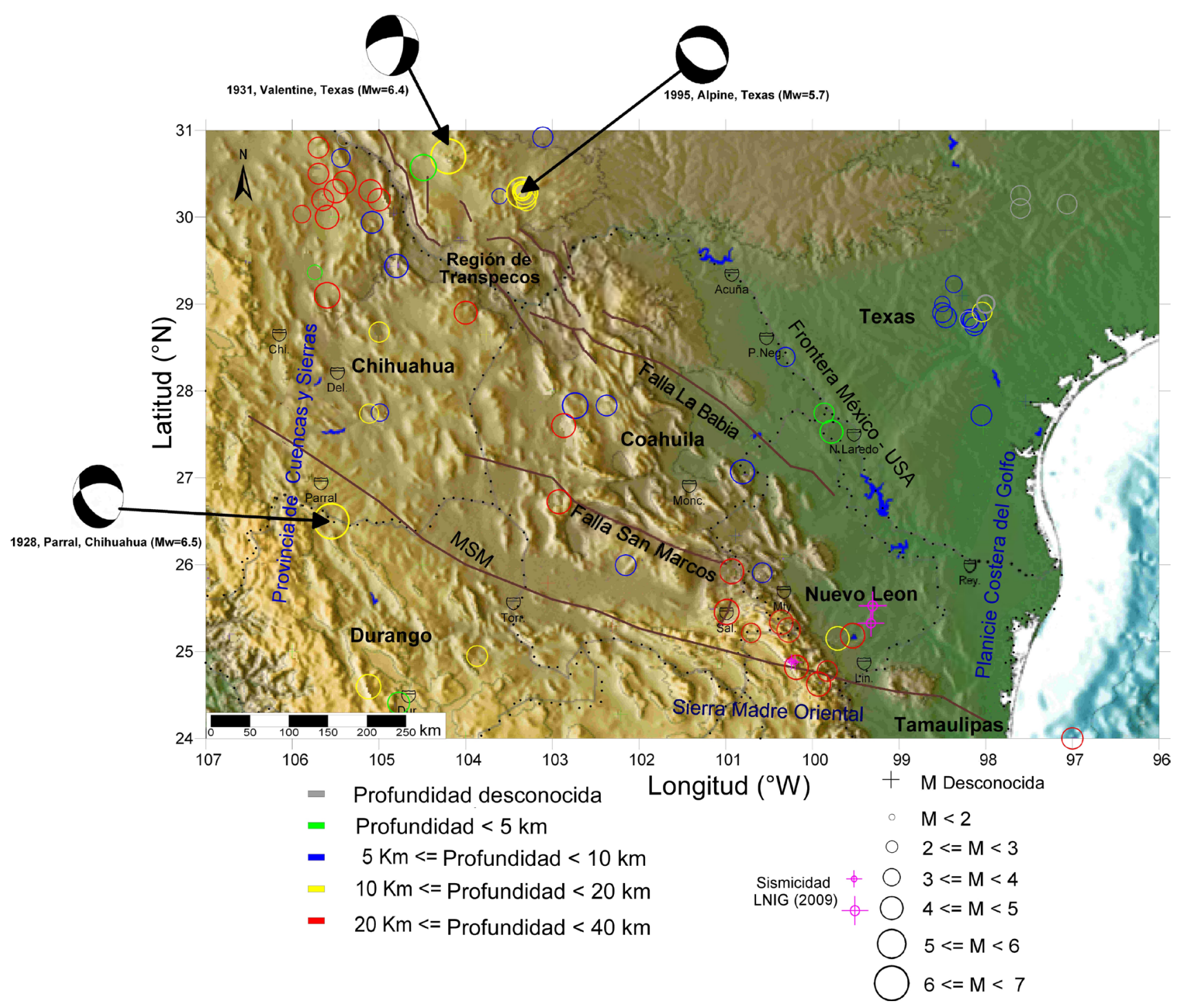

Figura 1. Sismicidad histórica (periodo 1847 - 2005) y reciente (2009) del noreste de México y sur de Texas (1928 - 2004), que incluye los principales lineamientos y fallas que han sido identificados o postulados en esta región. M representa la magnitud local. Se señala la ubicación de los principales centros de población (Chi: Chihuahua; Del: Delicias; Dur: Durango; Tor: Torreón; Mon: Monclova; Sal: Saltillo; Mty: Monterrey; Lin: Linares; P. Neg: Piedras Negras; Rey: Reynosa). Modificado de Galván-Ramírez y Montalvo-Arrieta (2008). 
acuerdo con la crónica mencionada en García Acosta y Suárez Reynoso (1996): “...en varias haciendas y ranchos de esta comprensión han caido algunas fincas y otras se han cuarteado, quedando casi inútiles..."

\subsection{Sismicidad reciente}

\subsubsection{Sismo del 14 de junio de $2009\left(M_{w}=5.0 ; H=20\right.$ $\mathrm{km}$; SSN-CMT)}

Este sismo fue localizado a $40 \mathrm{~km}$ al ENE de General Terán (Servicio Sismológico Nacional). De acuerdo con los reportes de los medios de comunicación, se sintió en los municipios de Allende, General Terán y Montemorelos, siendo estos dos últimos donde la sacudida del terreno fue más severa. No se reportaron daños a estructuras civiles.

Para la región de Montemorelos - General Terán, existe evidencia de sismicidad histórica. García Acosta y Suárez Reynoso (1996) reportaron al menos cuatro temblores (30/08/1838; 24/10/1909; 21/10/1911 y 03/12/1911) sentidos en la región de Montemorelos y localidades cercanas. Para los últimos dos eventos, las crónicas reportan intensidades sísmicas de III (Escala de CANCANI) en Montemorelos. Rodríguez Cabo (1946) estudió un enjambre de pequeños sismos en 1944. Este autor propuso, como fuente de estos sismos, al colapso de cavernas. Para esta misma región, el SSN ubicó un evento de magnitud 4.5 con una profundidad de $38 \mathrm{~km}$ al oeste de Montemorelos, ocurrido el 06/04/2004.

\subsubsection{Sismos del 20 y 21 de junio de $2009(M=3.5,3.7 y$ 3.7; $S S N)$}

El 20 y 21 de junio de 2009, se registraron tres terremotos en la estación LNIG (ubicada en la Facultad de Ciencias de la Tierra, UANL) en Linares, los cuales fueron sentidos en varias localidades del municipio de Galeana. De acuerdo con Montalvo Arrieta (2009), se documentó la presencia de grietas en algunas viviendas de las localidades Lagunita (El Orito), La Manteca y San José de la Joya. Esta última población se localizó en el área epicentral de los sismos del 20 de junio y en donde se experimentaron los mayores daños a casas y la escuela primaria. Montalvo Arrieta (2009) mencionó que las intensidades sísmicas (escala de Mercalli Modificada) experimentadas en el área epicentral fueron de IV-V. Los daños en viviendas corresponden con estructuras del Tipo A (muros de mampostería en seco o con barro, adobe y tapial de acuerdo con la escala de IMM), también se reportaron grietas en escuelas y deformación de ventanales.

De acuerdo con Rodríguez Cabo (1946), García Acosta y Suárez Reynoso (1996), Galván-Ramírez y Montalvo-Arrieta (2008) y Montalvo Arrieta (2009), se ha documentado evidencia de sismicidad distribuida en todo el estado de Nuevo León, así como daños a estructuras civiles (históricas y recientes) para algunos de estos terremotos. A partir de esta evidencia, se justifica conocer y cuantificar la respuesta sísmica de sitio para el estado, así como el potencial de riesgo sísmico para la región.

\section{Marco Geológico}

La mayor parte de México es una amalgama de terrenos adheridos a Norteamérica. Según lo anterior, la evolución geológica del país ha estado controlada por la acreción de terrenos tectonoestratigráficos a través de límites transformantes o convergentes en diferentes periodos geológicos (Campa y Coney, 1983; Sedlock et al., 1993; Ortega-Gutiérrez et al., 1994).

El noreste de México ha sido afectado por numerosos eventos tectónicos, para los cuales existe una documentación extensa en la literatura. Las rocas más antiguas identificadas corresponden a granulitas, cuarcitas y gneises, que han sido reconocidas en pozos perforados al este del anticlinorio Huizachal-Peregrina. A estas rocas, con base en datos geocronológicos, se les ha asignado una edad Grenvilliana (Garrison et al., 1980; Woods et al., 1991; RamírezRamírez, 1992) y cuyo origen pudo haber sido el oeste de los Estados Unidos, experimentando, durante el Paleozoico Tardío, un desplazamiento hasta su posición actual (OrtegaGutiérrez et al., 1995).

El Mesozoico marcó el inicio de los dos eventos tectónicos más importantes en el Noreste de México: (1) la separación del supercontinente Pangea y el subsecuente rift que permitió la apertura del Golfo de México (Buffler y Sawyer, 1985; Salvador, 1987; Wilson, 1990), y (2) la subducción de la Placa Farallón en el Oeste de México (Coney, 1978; Dickinson, 1981; Damon y Shafiqullah, 1991; Grajales-Nishimura et al., 1992). Entre el Jurásico y el Terciario, ocurrió la sedimentación de la secuencia carbonatada y siliciclástica que dio lugar a la columna tipo del Noreste de México. A partir de finales del Cretácico, la secuencia marina mesozoica fue plegada y fallada durante la orogenia Laramide (Padilla y Sánchez, 1982; 1986).

Los procesos geológicos mencionados han condicionado que la columna estratigráfica para esta porción del país, esté constituida por una secuencia de rocas con edades que cubren desde el Precámbrico hasta el Cuaternario (Figura 2), siendo la Sierra Madre Oriental (SMO), el rasgo más predominante. La SMO atraviesa el estado de Nuevo León y está constituida en su mayor parte por rocas sedimentarias terrígenas y carbonatadas del Mesozoico (Padilla y Sánchez, 1982, 1985; Eguiluz de Antuñano et al., 2000) depositadas en un ambiente de rift a margen pasivo, producto de una gran trasgresión marina iniciada desde el Calloviano-Oxfordiano (164-155 Ma; Götte, 1990; Goldhammer, 1999), sobre un basamento precámbrico y paleozoico, principalmente metamórfico pero también sedimentario. La secuencia sedimentaria del Terciario localizada en la Cuenca de Burgos, representa la extensión sur de la ensenada del Río Grande en Texas, EE.UU. (López-Ramos, 1983; Bryant et al., 1991). En la Cuenca de Burgos se depositó una potente secuencia arcillo-arenosa, con intercalación variable de areniscas de espesor delgado a medio. Esta se localiza sobre una amplia y extensa plataforma continental de bajo relieve 
y lenta subsidencia, en la que predominaron ambientes mixtos y marinos internos a medios. La sedimentación se efectuó en depósitos cíclicos sucesivos transgresivosregresivos del Paleoceno al Reciente y, que en general, representan una extensa progradación hacia el oriente (Echánove, 1986).

\section{Definición del mapa de las condiciones sísmicas de sitio a partir de mapas geológicos}

Para la creación de mapas regionales de las condiciones sísmicas de sitio, es necesario contar con una base de información geológica, para correlacionar con parámetros dinámicos de la respuesta sísmica de sitio, por ejemplo, velocidades de ondas de corte en términos de $\mathrm{V}_{\mathrm{S} 30}$. Los primeros trabajos que presentan correlaciones entre geología y la respuesta sísmica de sitio corresponden a Petersen et al. (1997). Estos autores, a partir de información geológica, generaron un mapa de las condiciones de sitio para la región de Los Ángeles, California, con la finalidad de realizar análisis probabilísticos de movimientos fuertes del terreno. Ellos clasificaron, de manera general, las unidades litológicas en tres tipos: roca dura (basamento), rocas suaves y aluvión. A esta clasificación le asignaron valores promedio de velocidades de propagación de ondas de corte definidas por Tinsley y Fumal (1985).

Otro tipo de clasificaciones han sido aplicadas por Park y Elrick (1998). Estos autores definieron los materiales geológicos que afloran en el sur de California, a partir de la edad de las diferentes unidades litológicas, asumiendo que existe una correlación aproximada en términos de las condiciones sísmicas de sitio. De manera que las unidades del Cuaternario las asocian con aluvión, el Terciario queda expresado como rocas suaves y al Mesozoico corresponden las rocas duras o de basamento (CTM). Park y Elrick (1998) correlacionaron esta clasificación de edades con perfiles sísmicos de ondas de corte $\left(\mathrm{V}_{\mathrm{S}}\right)$ y $\mathrm{V}_{\mathrm{S} 30}$. Ellos encontraron que los valores de $\mathrm{V}_{\mathrm{S} 30}$ varían con la edad y el tamaño de grano de las unidades litológicas, así que propusieron utilizar una clasificación más amplia que CTM. En este sentido, una manera para determinar la respuesta sísmica de sitio, en regiones con poca o nula instrumentación sísmica (sismómetros y/o acelerómetros), es mediante la estimación de las velocidades de propagación de ondas de corte de los sedimentos someros, utilizando métodos de exploración sísmica tales como la refracción. De manera general, se acepta que el promedio de $\mathrm{V}_{\mathrm{s}}$ para los primeros $30 \mathrm{~m}$ del subsuelo $\left(\mathrm{V}_{\mathrm{S} 30}\right)$ es un parámetro para predecir el potencial de amplificación de la sacudida sísmica (Holzer et al., 2005):

$$
V_{S 30}=\frac{\sum_{i=1}^{n} d_{i}}{\sum_{i=1}^{n} \frac{d_{i}}{V_{S i}}} \quad \text { Ecuación } 1
$$

donde: $\mathrm{d}_{\mathrm{i}}$ es el espesor de la $i$-ésima capa entre 0 y 30 $\mathrm{m} ; \mathrm{V}_{\mathrm{Si}}$ es el promedio de velocidad de ondas de corte en la $i$-ésima capa. La disminución del valor de $\mathrm{V}_{\mathrm{S} 30}$, en depósitos sedimentarios no consolidados del Cuaternario, se correlaciona con un incremento en la amplificación del movimiento del terreno durante terremotos (Williams et al., 2003).

De acuerdo con la NEHRP, las condiciones locales de la respuesta sísmica de sitio se pueden clasificar en seis diferentes grupos (Tabla 1). Holzer et al. (2005) mencionaron que esta clasificación es ampliamente usada en los EE.UU. y ha sido incorporada en diferentes códigos de construcción aplicados en diferentes países (Boore et al., 1994; Borcherdt, 1994; BSSC, 1994; Midorikawa et al., 1994). En este trabajo, la clasificación de la Tabla 1 y su correlación con los mapas geológicos escala 1:250000 del estado, han sido utilizados como un indicador de la respuesta sísmica de sitio, tomando como partida los estudios de Park y Elrick (1998) y Wills et al. (2000).

En el presente estudio, para realizar la correlación entre geología y velocidades de propagación de ondas sísmicas, se agruparon en primera instancia las unidades litológicas con edades y propiedades similares. Se utilizó el concepto de formación geológica, que de acuerdo con la definición de la Royal Geological and Mining Society of the Netherlands (Visser, 1980) es: (a) cualquier capa sedimentaria o serie consecutiva de capas suficientemente homogéneas o distintivas para ser consideradas como una unidad; (b) que pueda ser cartografiada y descrita en una sección geológica. Para el caso de sedimentos recientes, los cuales no han sido definidos bajo el concepto de formación, se utilizó el siguiente criterio: se clasificaron a partir de su edad y tipo de material, por ejemplo: Qal, aluvión del Cuaternario, o Tcg, conglomerado del Terciario. El siguiente punto para llevar a cabo la correlación entre geología y velocidades de propagación de ondas sísmicas fue la elección de la escala a utilizar, como refiere Wills et al. (2000). Si un mapa geológico puede ser usado para presentar la distribución de velocidades de ondas sísmicas, la pregunta que hay que resolver es: ¿cuál es el más adecuado? De manera general, se buscaron mapas geológicos que presentaran detalles que incluyeran edades, litologías y otros factores que influyen en la velocidad de ondas sísmicas, de manera particular

Tabla 1. Clasificación de suelos de acuerdo a la NEHRP*

\begin{tabular}{ccc}
\hline Tipo de suelo & Clasificación Roca/Suelo & $\boldsymbol{V} \boldsymbol{s 3 0}(\mathbf{m} / \mathbf{s})$ \\
\hline A & Roca dura & $>1500$ \\
B & Roca & $760-1500$ \\
C & Suelo muy denso o roca suave & $360-760$ \\
D & Suelo duro & $180-360$ \\
E & Suelo suave & $<180$ \\
F & Suelos especiales que requieren \\
& evaluación específica de sitio \\
\hline
\end{tabular}

*NEHRP: National Earthquake Hazard Reduction Program 
ondas de corte. A nivel nacional y regional, los mapas que cuentan con mayor detalle y que son accesibles para cualquier usuario son los que genera el INEGI a escala 1:50000. Existen mapas con mayor resolución, sin embargo son escasos y no tienen coberturas regionales. Para el estado de Nuevo León no existe una cobertura del $100 \%$ a escala 1:50000. De manera que, no se utilizó esta escala para generar el mapa de la distribución de velocidades para el estado. La siguiente escala utilizable, y para la cual si se cuenta con una cobertura del $100 \%$ y con disponibilidad, corresponde a la escala 1:250000. Esta escala aún cuenta con suficiente detalle para mostrar los rasgos geológicolitológicos regionales más significativos para el propósito de este trabajo. La información geológica utilizada para la generación de los mapas de la distribución de las unidades litológicas corresponde a los datos digitales del INEGI (2006), de acuerdo a las cartas geológicas F1401, F1402, G1401, G1402, G1404, G1405, G1407, G1408, G1410 y G1411 escala 1:250000, que cubren todo el estado de Nuevo León. La Figura 3 muestra el mapa geológico del área de estudio; en la Tabla 2 se describen las localizaciones, coordenadas y tipo de litología sobre la cual se realizaron los perfiles sísmicos. La verificación en campo se llevó a cabo a partir de seleccionar sitios característicos de afloramientos para cada una de las formaciones que conforman la columna litológica tipo para el estado de Nuevo León, donde se realizaron perfiles de refracción sísmica, con longitudes de acuerdo con las dimensiones del afloramiento. La columna estratigráfica compuesta (Figura 2) para esta porción del país se obtuvo a partir de Gursky (1996), quién definió la columna estratigráfica para el Anticlinorio HuizachalPeregrina (Paleozoico) y que corresponde al basamento en la región; Michalzik (1988) definió la columna estratigráfica para la secuencia siliciclástica y carbonatada del Mesozoico de la SMO, y Echánove (1986) describió la columna de la Provincia Cuenca de Burgos de edad terciaria, la cual se

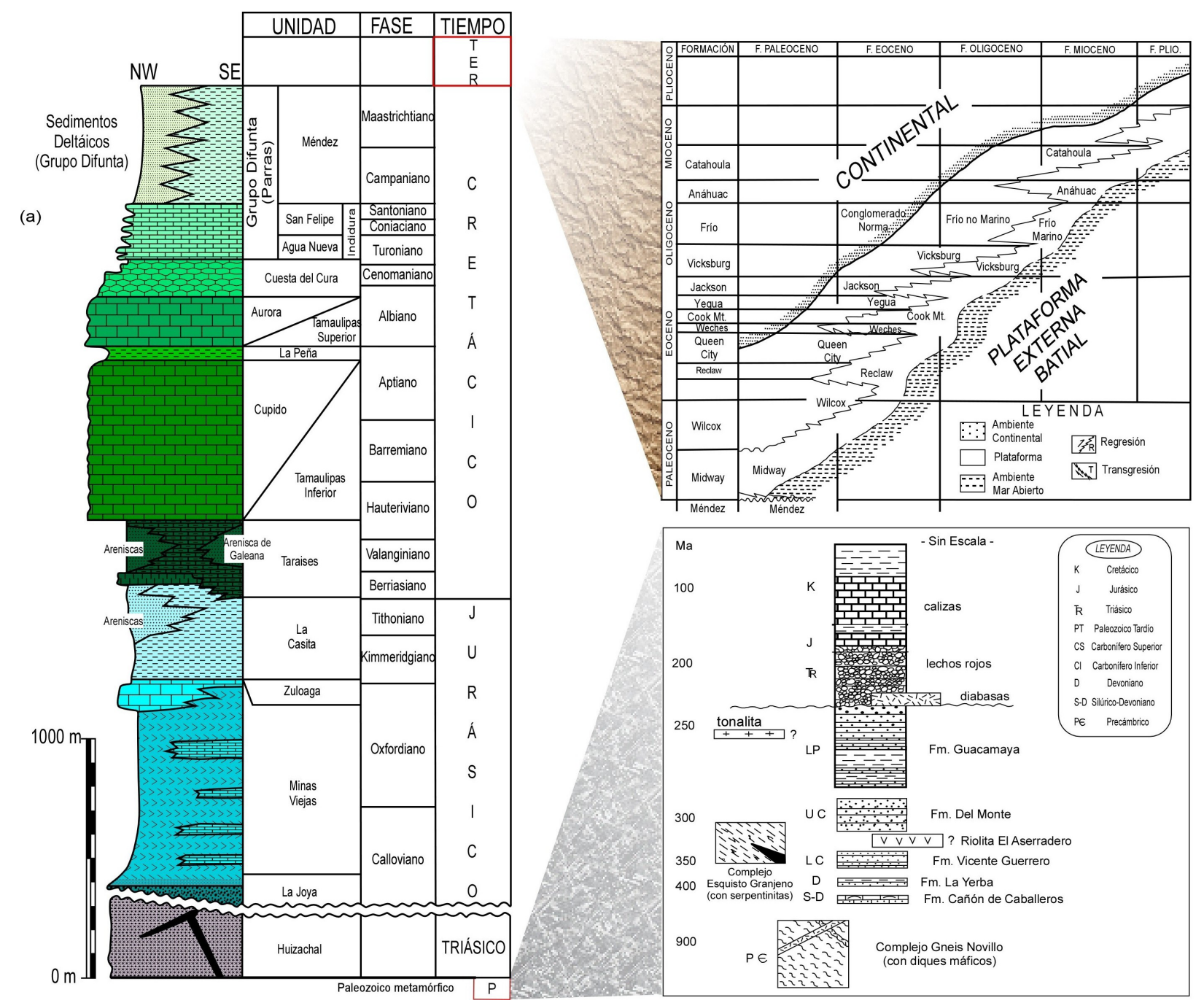

(b)

(c)

Figura 2. Columna estratigráfica compuesta del área de estudio. (a) Columna estratigráfica de la Sierra Madre Oriental (Michalzik, 1988); (b) Columna estratigráfica del Terciario, Provincia Cuenca de Burgos (Echánove, 1986); (c) Columna estratigráfica del Anticlinorio Huizachal-Peregrina (Gursky, 1996). 


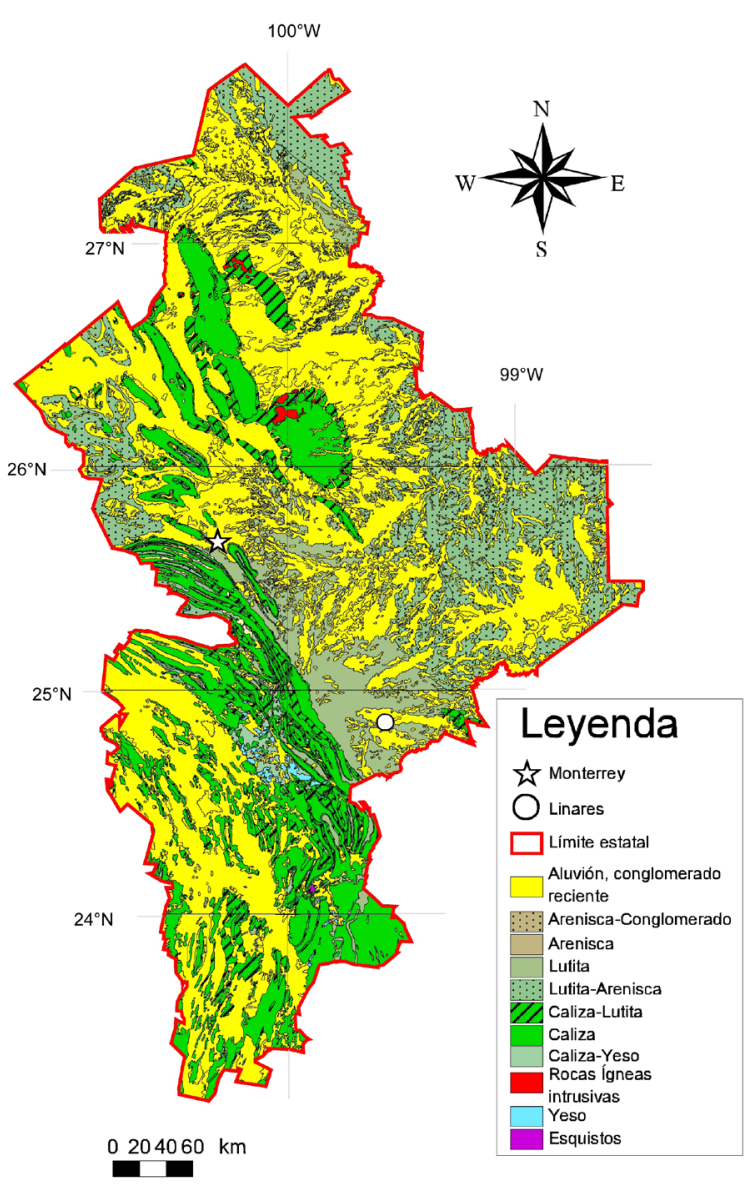

Figura 3. Mapa geológico del estado de Nuevo León.

ubica en la Planicie Costera del Golfo.

Algunos de los perfiles sísmicos se realizaron en los estados de Tamaulipas y Coahuila, como se mencionó anteriormente, donde se encuentran afloramientos tipo tanto para rocas que componen el basamento, como para formaciones del Terciario. En la Tabla 2 se presentan las coordenadas, localidades y descripción del tipo de litología de los sitios. En la realización de los perfiles sísmicos, también se consideraron las variaciones en los ambientes de depositación de algunas formaciones, para determinar el valor promedio de la formación. Tal es el caso, por ejemplo, de la Formación Tamaulipas Superior, equivalente de cuenca de la Formación Aurora; la Formación Cupido, equivalente de plataforma de la Formación Tamaulipas Inferior; la Formación La Casita, con un miembro lutítico y otro compuesto por intercalaciones de lutitas, calizas y areniscas; la Formación Taraises, con un miembro inferior de caliza arcillosa y uno superior de igual composición, que hacia la cima se vuelve más calcáreo. En las zonas urbanas de Linares y Monterrey, se contó además con información litológica obtenida a partir de perforaciones, lo que permitió realizar correlaciones de la distribución de las velocidades con respecto a las unidades encontradas a profundidad. Esta
Tabla 2. Ubicación de sitios en donde se realizaron los perfiles sísmicos.

\begin{tabular}{|c|c|}
\hline Sitio o perfil & Latitud $\left({ }^{\circ} \mathrm{N}\right)$ \\
\hline Allende & 25.33333 \\
\hline Balastre, Linares & 25.16389 \\
\hline Colonia Camachito, Linares & 24.86200 \\
\hline Colonia Camachitol, Linares & 24.86200 \\
\hline Ejido 5 Señores & 24.87500 \\
\hline Ejido La Escondida & 24.91056 \\
\hline Facultad de Ciencias de la Tierra, Linares & 24.89722 \\
\hline Facultad de Ciencias Forestales, Linares & 24.79055 \\
\hline Cañón la Boca, Santiago & 25.43867 \\
\hline Cañón la Boca, Santiago & 25.43591 \\
\hline Guadalupe La Joya & 25.28889 \\
\hline Colonia Industrial, Linares & 24.88583 \\
\hline Sierra de las Mitras, Monterrey & 24.67861 \\
\hline $\begin{array}{l}\text { Colonia La Petaca, Linares } \\
\text { Cina }\end{array}$ & 24.86167 \\
\hline Colonia Provileón, Linares & 24.85917 \\
\hline Colonia San Fco., Linares & 24.85833 \\
\hline Colonia El Nogalar, Linares & 24.83828 \\
\hline Colonia Carolinas, Linares & 24.87725 \\
\hline Panteón Municipal, Linares & 24.86850 \\
\hline Ejido San Lázaro, Linares & 24.56917 \\
\hline Ejido San Pablo de Tranquitas, Galeana & 24.69639 \\
\hline San Pablo de Tranquitas, Galeana & 24.69639 \\
\hline Ejido La Huiche, Galeana & 24.69305 \\
\hline Ejido Santa Rosa, Iturbide & 24.71889 \\
\hline Ejido Santa Rosa, Iturbide & 24.69695 \\
\hline Ejido Santa Rosa, Iturbide & 24.69389 \\
\hline Ejido Santa Rosa, Iturbide & 24.69389 \\
\hline Carr. Rumbo Nuevo-Cd. Victoria & 23.58972 \\
\hline Carr. Rumbo Nuevo-Cd. Victoria & 23.58972 \\
\hline Carr. Rumbo Nuevo-Cd. Victoria & 23.58944 \\
\hline Carr. Rumbo Nuevo-Cd. Victoria & 23.58639 \\
\hline Carr. Rumbo Nuevo - Cd. Victoria & 23.58972 \\
\hline Rio Santa Catarina, Monterrey & 25.66472 \\
\hline Monterrey & 25.69736 \\
\hline Monterrey & 25.69686 \\
\hline Monterrey & 25.69764 \\
\hline Monterrey & 25.69782 \\
\hline Monterrey & 25.69611 \\
\hline Monterrey & 25.69617 \\
\hline Monterrey & 25.69745 \\
\hline Monterrey & 25.69760 \\
\hline Cd. Deportiva, Monterrey & 25.68770 \\
\hline Hospital Sta. Engracia, Monterrey & 25.64340 \\
\hline Gigante, Garza Sada, Monterrey & 25.66130 \\
\hline HEB - Cumbres, Monterrey & 25.73080 \\
\hline Hospital Universitario, Monterrey & 25.69180 \\
\hline Estación Eléctrica, Monterrey & 25.61310 \\
\hline Inst. de Ingenieria Civil, Monterrey & 25.73350 \\
\hline Lago, Monterrey & 25.78490 \\
\hline Cerro de las Mitras, Monterrey & 25.67800 \\
\hline Parque Aztlán, Monterrey & 25.76280 \\
\hline Col. Solidaridad, Monterrey & 25.76070 \\
\hline Parque España, Monterrey & 25.73340 \\
\hline Fraccionamiento los Pinos, Monterrey & 25.61880 \\
\hline Prepa ITESM, Monterrey & 25.74050 \\
\hline Col. Sta. Isabel, Monterrey & 25.77600 \\
\hline Río Santa Catarina, Monterrey & 25.66460 \\
\hline Col. Roma, Monterrey & 25.65300 \\
\hline Parque de Beisbol, Col. Roma, Mty & 25.64990 \\
\hline Ruiz Cortinez, Monterrey & 25.73130 \\
\hline 7 Cerros, Monterrey & 25.74500 \\
\hline Agencia Toyota, Monterrey & 25.63900 \\
\hline UANL, CU., Monterrey & 25.72190 \\
\hline $\begin{array}{l}\text { Mina El Relinche, Cañón Novillo, } \\
\text { Tamaulipas }\end{array}$ & 23.71359 \\
\hline Cañón Novillo, Tamaulipas & 23.71758 \\
\hline Cañón Novillo, Tamaulipas & 23.72306 \\
\hline Cañón Novillo, Tamaulipas & 23.71824 \\
\hline $\begin{array}{l}\text { Carr. Reynosa - Nvo. Padilla, Cuenca de } \\
\text { Burgos }\end{array}$ & 24.28182 \\
\hline $\begin{array}{l}\text { Carr. Reynosa - Nvo. Padilla, Cuenca de } \\
\text { Burgos }\end{array}$ & 24.27937 \\
\hline $\begin{array}{l}\text { Carr. Cd. Victoria-Matamoros, Cuenca de } \\
\text { Burgos }\end{array}$ & 24.45995 \\
\hline $\begin{array}{l}\text { Carr. Cd. Victoria-Matamoros, Cuenca de } \\
\text { Burgos }\end{array}$ & 24.73477 \\
\hline $\begin{array}{l}\text { Carr. Cd. Victoria-Matamoros, Cuenca de } \\
\text { Burgos }\end{array}$ & 24.50753 \\
\hline Sierra de San Carlos, Tamaulipas & 24.61899 \\
\hline $\begin{array}{l}\text { Rincón Murillo, Sierra de San Carlos, } \\
\text { Tamaulipas }\end{array}$ & 24.52732 \\
\hline $\begin{array}{l}\text { Derrames Basálticos, Sierra de San Carlos, } \\
\text { Tamaulipas }\end{array}$ & 24.64459 \\
\hline Cañón La Peregrina, Tamaulipas & 23.77178 \\
\hline Cañón La Peregrina, Tamaulipas & 23.77235 \\
\hline Cañón La Peregrina, Tamaulipas & 23.77127 \\
\hline Cañón La Peregrina, Tamaulipas & 23.77202 \\
\hline Cañón La Peregrina, Tamaulipas & 23.77191 \\
\hline Cañón La Boca, Santiago & 25.43904 \\
\hline Cañón La Boca, Santiago. & 25.43893 \\
\hline Cañón La Boca, Santiago & 26.43851 \\
\hline Cañón La Boca, Santiago. & 25.43459 \\
\hline Cañón La Huasteca, Monterrey & 25.64805 \\
\hline Cañón La Huasteca, Monterrey & 25.64805 \\
\hline Cañón La Huasteca, Monterrey & 25.63470 \\
\hline Cañón La Huasteca, Monterrey & 25.63662 \\
\hline $\begin{array}{l}\text { Carricitos, Cuenca La Popa, Sinclinal } \\
\text { Delgado }\end{array}$ & 26.02726 \\
\hline $\begin{array}{l}\text { Delgado } \\
\text { Cuenca La Popa, Lentil El Gordo }\end{array}$ & 26.02874 \\
\hline $\begin{array}{l}\text { Cuenca La Popa, Sinclinal Delgado } \\
\text { (Núcleo) }\end{array}$ & 26.02302 \\
\hline $\begin{array}{l}\text { (Núcleo) } \\
\text { El Delgado, Cuenca La Popa }\end{array}$ & 25.99143 \\
\hline Cuenca La Popa & 25.98052 \\
\hline & 25.94989 \\
\hline Cuenca La Popa & 25.93885 \\
\hline Paredón, Coahuila & 25.93863 \\
\hline
\end{tabular}


correlación ha quedado descrita por Montalvo-Arrieta et al. (2005, 2008). Estos autores compararon las estructuras de velocidades de ondas $P$ y $S$ obtenidas, con espesores y tipo de litología de pozos localizados en las zonas urbanas de Linares y Monterrey, encontrando que existe una fuerte correlación entre los cambios litológicos y las velocidades de ondas sísmicas. Por otro lado, Infante et al. (2010) han correlacionado información geotécnica de pozos someros (litología, espesores, porcentaje de humedad y granulometría) con información generada de la inversión conjunta del gradiente cruzado de datos de resistividad y velocidad de ondas $P$. Estos autores han definido la geometría y la clasificación de las unidades litológicas en un sitio de pruebas, ubicado en la Facultad de Ciencias de la Tierra de la UANL (Linares). Los valores obtenidos para ondas $P$ y su correlación con las unidades litológicas en esa área presentan similitud con lo observado en este trabajo, para cambios litológicos donde se ha considerado al aluvión con la lutita de la Formación Méndez. De esta forma, a partir de los perfiles sísmicos realizados, se agruparon las diferentes unidades geológicas que tienen valores similares de $\mathrm{V}_{\mathrm{S} 30}$, a fin de generar el primer mapa de las condiciones sísmicas de sitio, basado en valores de $\mathrm{V}_{\mathrm{S} 30}$ y rasgos geológicos. Es claro que, los resultados de las condiciones sísmicas de sitio obtenidos en este estudio, seguirán actualizándose conforme se incremente la base de datos de $\mathrm{V}_{\mathrm{S} 30} \mathrm{y}$ de información geológica detallada de sitios específicos.

\section{Asignación de las unidades geológicas a la clasificación de $\mathbf{V}_{\mathrm{S} 30}$}

Considerando los estudios de Park y Elrick (1998) y Wills et al. (2000), para realizar la correlación entre materiales geológicos y velocidades de propagación de ondas de corte, se llevó a cabo la asignación de los valores de $\mathrm{V}_{\mathrm{S} 30}$ a las unidades litológicas que afloran en el área de estudio. De acuerdo con los valores promedio obtenidos en campo para cada formación se asignaron estos a la unidad litológica correspondiente para generar la primera aproximación de la distribución de valores de $\mathrm{V}_{\mathrm{S} 30}$ en el estado. Como se mencionó con anterioridad, se utilizó el concepto de formación para la clasificación de las unidades geológicas. Inicialmente se correlacionaron las formaciones y los valores de $\mathrm{V}_{\mathrm{S} 30}$ de acuerdo con las compilaciones realizadas por Montalvo-Arrieta et al. $(2005,2008)$ para las zonas urbanas de Linares y Monterrey, respectivamente. Para estos trabajos sólo se contó con información de algunas formaciones del Mesozoico, que están compuestas por calizas y lutitas, así como material aluvial del Terciario y Cuaternario. La relación de valores de $\mathrm{V}_{\mathrm{S} 30}$ obtenidos se correlaciona de la siguiente manera: (a) material aluvial con valores promedio de $\mathrm{V}_{\mathrm{S}}$ de $632 \mathrm{~m} / \mathrm{s}$; (b) conglomerados del Terciario con velocidades promedio de $\mathrm{V}_{\mathrm{S}}$ de $1370 \mathrm{~m} / \mathrm{s}$; (c) lutitas de la Formación Méndez con valores de $\mathrm{V}_{\mathrm{S}}$ de 1774 $\mathrm{m} / \mathrm{s}$; y (d) calizas de edad mesozoica con valores de $\mathrm{V}_{\mathrm{S}}$ de $2311 \mathrm{~m} / \mathrm{s}$. De este primer análisis, se encontró que existe una correlación aproximada en términos de las condiciones sísmicas de sitio y la edad, composición y velocidad. Así, las unidades del Cuaternario (aluvión) se asocian con velocidades bajas correlacionadas con sitios clase C (Tabla 1), los conglomerados cementados del Terciario quedan definidos como sitios clase B y los sedimentos de edad mesozoica (formaciones Méndez, San Felipe, Agua Nueva y Cuesta del Cura) corresponden a rocas duras (sitios clase A). Para el resto de las formaciones geológicas de edad Paleozoico, Mesozoico y Terciario, el tipo de clasificación de acuerdo con la Tabla 1, se obtuvo a partir de los perfiles sísmicos de refracción efectuados en este estudio.

\section{Perfiles sísmicos de refracción y estimación de los valores de $V_{S 30}$}

La determinación de la estructura de velocidades para cada sitio de medición se llevó a cabo mediante perfiles sísmicos de refracción, los cuales fueron interpretados a partir de curvas de tiempo de viaje (método de intercepción de pendientes). Para esto, se seleccionaron los primeros arribos de las fases identificadas, asumiendo que fueron refractadas en la misma interfase. El valor de la velocidad se obtuvo a partir de las pendientes que identifican a los arribos, donde se asume que la velocidad permanece constante para cada estrato. El equipo para la realización de los perfiles sísmicos de refracción consistió de un Remote Acquisition System (RAS-24), con 24 bits para el convertidor A/D y 24 canales. Se utilizaron geófonos de componente horizontal y vertical con frecuencia natural de $28 \mathrm{~Hz}$ para la determinación de las velocidades de propagación de ondas $P$ y $S$. La fuente utilizada para la generación de la perturbación elástica que se propagó a través del subsuelo fue un marro con un peso de $4.5 \mathrm{~kg}$. El arreglo de geófonos fue variable y estuvo condicionado con la longitud del afloramiento. Los perfiles de refracción sísmica se llevaron a cabo en terracerías y/o caminos vecinales (horizontales o semi-horizontales) que cortaron los afloramientos de interés para las áreas no urbanas. Mientras que, en las ciudades éstos se realizaron en parques, terrenos baldíos, áreas residenciales, públicas o industriales, como ha sido descrito en Montalvo-Arrieta et al. $(2005,2008)$ e Infante et al. (2010). Para este trabajo se realizaron 708 perfiles sísmicos de refracción distribuidos en diferentes localidades del área de estudio. Estos perfiles cubrieron las diferentes formaciones que conforman la columna estratigráfica (Figura 2), e incluyen a los perfiles sísmicos obtenidos en las zonas urbanas de Linares y Monterrey. La Tabla 3 muestra los materiales y localidades donde se realizaron los perfiles de refracción sísmica, las formaciones estudiadas y su clasificación sísmica asignada de acuerdo con la Tabla 1. A continuación se presentan los valores obtenidos para algunos de los perfiles sísmicos de refracción, en sitios 
Tabla 3. Materiales geológicos, sitios donde se realizaron los perfiles de refracción sísmica y su clasificación de acuerdo con la Tabla 1.

\begin{tabular}{|c|c|c|c|c|c|c|}
\hline \multirow[b]{2}{*}{ Edad } & \multirow[b]{2}{*}{ Formación } & \multirow[b]{2}{*}{ Litología } & \multirow[b]{2}{*}{ Símbolo } & \multicolumn{3}{|c|}{ Clasificación NEHRP y localidad donde se obtuvieron los perfiles } \\
\hline & & & & A & B & C \\
\hline $\mathbf{Q}$ & Aluvión y limos del Cuaternario & Aluvión - Limos y Suelos & Qhoal-L-S & & & Mty, Ln \\
\hline $\mathbf{T}$ & Conglomerado & & TCg & & Mty, Ln & \\
\hline Ple & Calizas Lacustres & Calizas Lacustres & $\mathrm{TCz}$ & & $\mathrm{R}-\mathrm{NP}$ & \\
\hline Mio & Basaltos & Basaltos & TmBs & $\mathrm{SSC}$ & & $\mathrm{SSC}$ \\
\hline ol & Gabros & Gabros & ToGa & $\mathrm{sSC}$ & & \\
\hline OI & Sienitas & Sienitas & ToSi & SSC & & \\
\hline Plio & Cong. Anáhuac & Conglomerado & $\mathrm{TpCg}$ & & $\mathrm{V}-\mathrm{M}$ & $\mathrm{V}-\mathrm{M}$ \\
\hline ol & Frío & Lutitas -Areniscas & To $\quad \mathrm{Lu}-\mathrm{Ar}$ & & & $\mathrm{JF}$ \\
\hline ol & Vicksburg & Areniscas - Lutitas & ToAr $-\mathrm{Lu}$ & & EZ & $\mathrm{EZ}$ \\
\hline Em & Viento & Areniscas - Limolitas & Tem $\quad \operatorname{Ar}-\mathrm{Lm}$ & $\mathrm{Crc}$ & & \\
\hline $\mathbf{E i}$ & Adjuntas & Areniscas - Limolitas rojas & $\begin{array}{c}\mathrm{Tei} \\
\mathrm{Ar}-\mathrm{Lmr}\end{array}$ & & $\mathrm{Crc}$ & \\
\hline PIc-m-Ei & Wilcox & Sedimento arcillo-arenoso y arenisca & Tplc Arc-Arn-Ar & & Bal & Bal \\
\hline Plc-m & Midway & Sed. Arcillosos & TplcArc & GJ & & \\
\hline Plc & Velasco & Areniscas & TplcAr & & ET & \\
\hline Ks & Potrerillos & Areniscas & KsAr & & $\mathrm{Crc}$ & $\mathrm{Crc}$ \\
\hline Ks & Muerto & Areniscas-Lutitas & KsAr-Lu & & ED, $P$ & ED, P \\
\hline Ks & Méndez & Lutitas calcáreas & Ks Lu-cal & $\mathrm{Ln}, \mathrm{CB}$ & & \\
\hline Km-s & San Felipe & Caliza margosa & Kms Cz-mg & Mty, Ln, Al & & $\mathrm{Al}$ \\
\hline Ks & Agua Nueva & Caliza-Lutita carbonosa & Ks $\quad$ Cz-Lu-ca & $\mathrm{RN}-\mathrm{CV}, \mathrm{CB}$ & & \\
\hline $\mathbf{K m}$ & Cuesta del Cura & Caliza & $\mathrm{KmCz}$ & $\mathrm{RN}-\mathrm{CV}, \mathrm{CB}$ & & \\
\hline Km & Aurora/ Tamp. Sup. & Caliza & $\mathrm{KmCz}$ & $\mathrm{RN}-\mathrm{CV}$ & & \\
\hline $\mathbf{K i}$ & La Peña & Caliza - Lutita & KiCz-Lu & $\mathrm{SR}, \mathrm{CB}$ & & \\
\hline $\mathbf{K i}$ & Cupido/ Tamp. Inf. & Caliza & $\mathrm{KiCz}$ & $\mathrm{SR}, \mathrm{CB}, \mathrm{CH}$ & & \\
\hline $\mathbf{K i}$ & Taraises & Caliza-Lutita & KiCz-Lu & $\mathrm{SR}, \mathrm{CB}, \mathrm{CH}$ & & \\
\hline Js-Ki & Casita & Lutita-Caliza-Arenisca & JsKi Lu-Cz-Ar & & $\mathrm{SR}, \mathrm{CB}, \mathrm{CH}$ & \\
\hline Js & Zuloaga & Caliza & $\mathrm{JsCz}$ & $\mathrm{Gl}, \mathrm{CB}, \mathrm{CH}$ & & \\
\hline Jm-s & Minas Viejas & Evaporitas & JmsEv & & $\mathrm{Crc}, \mathrm{Gl}$ & \\
\hline TR-J & Huizachal & Lechos Rojos & TRJLR & & $\mathrm{Gl}$ & \\
\hline TR-J & Huizachal & Lavas-Riolitas-Piroclastos -Areniscas & $\begin{array}{c}\text { TRJ } \\
\text { Lv-Ri-P-Ar }\end{array}$ & & $\mathrm{RN}-\mathrm{CV}$ & \\
\hline Pz-Per & Guacamaya & Areniscas-Lutitas & PzpAr-Lu & $\mathrm{CP}$ & & \\
\hline Pz-Car & Vicente Guerrero & Areniscas-Lutitas & PzcAr-Lu & $\mathrm{CP}$ & & СР \\
\hline Pz-Sil & Cañón de Caballeros & Calizas-Lutitas-Areniscas & Pzs $\quad$ Cz-Lu-Ar & $\mathrm{CP}$ & & $\mathrm{CP}$ \\
\hline $\mathbf{P z}$ & Esquisto Granjeno ( $2^{a}$ secuencia) & Serpentinita & PzSerp & $\mathrm{CN}$ & & $\mathrm{CN}$ \\
\hline Pz & Esquisto Granjeno ( $\left(1^{a}\right.$ secuencia $)$ & Esquisto & PzE & $\mathrm{CN}$ & & $\mathrm{CN}$ \\
\hline $\operatorname{Pr}$ & Gneis Novillo & Gneis & PrGn & $\mathrm{CN}$ & & \\
\hline
\end{tabular}

NEHRP: National Earthquake Hazard Reduction Program. Nuevo León.- Mty: Monterrey; SSC: Sierra de San Carlos; Bal: Balastre; GJ: Gpe. La Joya; ED: El Delgado; Crc: Carricitos; Ln: Linares; Al: Allende; SR: Santa Rosa; Gl: Galeana; CB: Cañón la Boca; CH: Cañón la Huasteca. Tamaulipas.- R-NP: Carr. Reynosa-Nuevo Padilla; V-M: Carr. Victoria-Matamoros; JF: Carr. Jiménez-San Fernando; ET: El Tinieblo; EZ: El Zacatal; RN-CV: Carr. Rumbo Nuevo-Cd. Victoria; CP: Cañón Peregrina; CN: Cañón Novillo. Coahuila.- P: Paredón. Q: Cuaternario; T: Terciario; Ple: Pleistoceno; Mio: Mioceno; Ol: Oligoceno; Plio: Plioceno; Em: Eoceno medio; Ei: Eoceno inferior; Plc-m-Ei: Paleoceno medio- Eoceno inferior; Plc-m: Paleoceno medio; Plc: Paleoceno; Ks: Cretácico. superior; Km-s; Cretácico medio-superior; Km: Cretácico medio; Ki Cretácico inferior; Js: Jurásico superior; Js-Ki: Jurásico superior-Cretácico inferior; Jm-s: Jurásico medio-superior; TR-J: Triásico-Jurásico; Pz-Per: Paleozoico-Pérmico; Pz-Car: Paleozoico-carbonífero; Pz-Sil: Paleozoico-silúrico; Pz: Paleozoico; Pr: Precámbrico. Los símbolos son los mismos que en la tabla 4.

característicos localizados en el área de estudio.

\subsection{Precámbrico y Paleozoico}

\section{1.1 Gneis Novillo (Precámbrico)}

El Precámbrico se encuentra definido por el Gneis Novillo (Muir, 1936; King, 1942; Flawn y Díaz-González., 1959; Fries et al., 1962, 1974; Denison et al., 1970; Cossío-Torres, 1988) y representa el basamento cristalino metamorfizado y está compuesto por varias unidades: Gneis Bandeado, Gneis Cataclástico, Gneis de Ojos o Augen Gneiss. El complejo metamórfico del Gneis Novillo tiene su localidad tipo en el Cañón Novillo o Río San Marcos a $8 \mathrm{~km}$ al SW de Ciudad Victoria, Tamaulipas dentro del núcleo del Anticlinorio Huizachal-Peregrina. A partir de los perfiles sísmicos realizados en esta localidad, los valores de las velocidades de ondas $P$ y $S$ fueron de $3550 \mathrm{~m} / \mathrm{s}$ y $2320 \mathrm{~m} / \mathrm{s}$, respectivamente. En total se realizaron 6 perfiles sísmicos obteniéndose un valor promedio de $\mathrm{V}_{\mathrm{S} 30}$ de 2105 $\mathrm{m} / \mathrm{s}$. En la Tabla 4 se presenta esta información junto con la desviación estándar, así como la relación $V S / V p$ para cada unidad litológica analizada. 


\subsection{Paleozoico}

\subsubsection{Esquisto Granjeno (Paleozoico Superior)}

Se encuentra presente en el Anticlinorio de HuizachalPeregrina y está conformado por dos secuencias: una metasedimentaria y una metamagmática. Los perfiles sísmicos realizados sobre la primer secuencia (formada a partir de un metamorfismo de bajo grado de sedimentos parcialmente ricos en arcillas; Castillo-Rodríguez, 1988) se localizan en el Cañón Novillo (Cd. Victoria, Tamaulipas), siendo las velocidades sísmicas obtenidas en este sitio, para ondas $P$ y $S$, de $3000 \mathrm{~m} / \mathrm{s}$ y $2080 \mathrm{~m} / \mathrm{s}$, respectivamente. La segunda de ellas (metamagmática) está conformada por serpentinitas (formada a partir de metamorfismo de bajo grado de basaltos o gabros; Salas-Pizá, 1970). Los valores de las velocidades de propagación de ondas $P$ y $S$ fueron de $4460 \mathrm{~m} / \mathrm{s}$ y $3110 \mathrm{~m} / \mathrm{s}$, respectivamente. En total se llevaron a cabo 4 perfiles sísmicos, dos en la secuencia metasedimentaria y los otros dos en la metamagmática. Debido a las diferencias presentes en composición de estas unidades del Paleozoico, se obtuvieron valores separados de $V_{\text {S30 }}$, los cuales se describen en la Tabla 4.

\section{2. 2 Formación Cañón de Caballeros (Silúrico)}

Esta formación contiene secuencias calcáreo-clásticas, con conglomerados y areniscas, que se localizan en el Cañón Peregrina, al oeste de Cd. Victoria, Tamaulipas, y sobreyace al Gneis Novillo (Gursky, 1996). Las velocidades de propagación de ondas $P$ y $S$ para ésta formación son de 3110 $\mathrm{m} / \mathrm{s}$ y $1860 \mathrm{~m} / \mathrm{s}$. Debido a la ausencia de más afloramientos accesibles, sólo se realizaron dos perfiles sísmicos y el valor promedio de ondas de corte obtenido es de $1840 \mathrm{~m} / \mathrm{s}$, el cual se reporta en la Tabla 4, junto con su desviación estándar y la relación $V s / V p$.

\subsubsection{Formación Vicente Guerrero (Mississípico Inferior)}

Conjunto constituido por areniscas cuarcíferas de color gris oscuro, con bandas conglomeráticas y lutitas negras (Gursky, 1996). Esta formación, descrita formalmente por Carrillo-Bravo (1961), tiene su localidad tipo en el Cañón de la Peregrina y se ha determinado que, donde se realizaron los perfiles sísmicos, tiene un espesor de $160 \mathrm{~m}$. Las velocidades de propagación de ondas $P$ y $S$ obtenidas son de $4360 \mathrm{~m} / \mathrm{s}$ y $2820 \mathrm{~m} / \mathrm{s}$, respectivamente. En esta formación se llevaron a cabo cuatro perfiles, cuyo valor promedio de $\mathrm{V}_{\mathrm{s}}=2560$ $\mathrm{m} / \mathrm{s}$ (ver Tabla 4).

\subsubsection{Formación Guacamaya (Pérmico)}

Está formación consiste de capas de areniscas gradadas e interestraficadas con limolitas y lutitas. Además se han descrito areniscas de origen volcánico y conglomerados. Los perfiles sísmicos se llevaron a cabo en el Cañón Peregrina. Esta formación es la más prominente del complejo sedimentario Paleozoico del Anticlinorio HuizachalPeregrina (Gursky, 1996). Los valores de velocidades sísmicas obtenidos para esta unidad son de $3910 \mathrm{~m} / \mathrm{s}$ para ondas $P$ y de $2430 \mathrm{~m} / \mathrm{s}$ para ondas $S$, con un valor promedio de ondas de corte de $2660 \mathrm{~m} / \mathrm{s}$.

\subsection{Mesozoico y Terciario}

Para las rocas del Mesozoico, los perfiles sísmicos de refracción realizados se ubicaron en varias localidades distribuidas en la SMO. Debido al grado de competencia de la mayor parte de las formaciones (Tabla 1), se escogieron caminos de terracería para colocar los geófonos. Para aquellas unidades donde existen cambios en las facies de sedimentación, como en las formaciones La Casita, Tamaulipas Inferior/Cupido, Tamaulipas Superior/Aurora, se eligieron diversos puntos de medición para valorar el efecto de las variaciones litológicas sobre las velocidades de propagación de las ondas sísmicas. Para tasar lo anterior, se realizaron mediciones a diferentes latitudes (sitios localizados tanto en el estado de Nuevo León como en Tamaulipas). Como se ha mencionado, la Tabla 2 muestra las coordenadas geográficas y localidades donde se llevaron a cabo los perfiles sísmicos de refracción. En las Tablas 3 y 4 se presentan la descripción de las formaciones y litologías analizadas, el número de perfiles realizados, el valor promedio de $\mathrm{V}_{\mathrm{S} 30}$ obtenido, así como su desviación estándar y su relación $V s / V p$ correspondiente.

De manera general, las formaciones del Mesozoico (Figura 2a) presentan valores de $\mathrm{V}_{\mathrm{S} 30}$ que corresponden a sitios clase $A\left(V_{S 30} \geq 1500 \mathrm{~m} / \mathrm{s}\right)$. Los perfiles realizados en cada una de estas formaciones presentan promedios de velocidad de ondas de corte que varían entre $1540 \mathrm{~m} / \mathrm{s}$, para areniscas bien consolidadas y conglomerados de la Formación Huizachal, localizados al sur de Galeana (que corresponden a la base de la columna mesozoica), hasta valores de $2149 \mathrm{~m} / \mathrm{s}$ para la lutita de la Formación Méndez, la cual se ubica en la cima de la secuencia sedimentaria del Mesozoico. Los valores máximos medidos se correlacionan con los paquetes masivos de calizas de las formaciones Cupido/Tamaulipas Inferior - Aurora/Tamaulipas Superior, así como la caliza margosa intercalada con lutita de la Formación San Felipe, la cual presenta valores promedio de ondas de corte de $2381 \mathrm{~m} / \mathrm{s}$. En la Tabla 4 se presenta todos los valores para las formaciones del Mesozoico, así como el número de perfiles realizados, su desviación estándar y relación $V s / V p$ obtenidos en el trabajo de campo. En la Tabla 3 se presenta su clasificación de acuerdo con $\mathrm{V}_{\mathrm{S} 30}$.

Por otro lado, se observó el papel importante del grado de consolidación de los materiales geológicos en la disminución de las velocidades de propagación de ondas sísmicas. Las formaciones del Terciario (Figura 2b) presentaron valores promedio de velocidades de propagación de ondas de corte menores que las rocas del Mesozoico y Paleozoico. Los valores de $\mathrm{V}_{\mathrm{S} 30}$ para rocas sedimentarias Terciarias se clasificaron como materiales tipo B y $\mathrm{C}$ de acuerdo con la Tabla 1. Las velocidades más bajas medidas corresponden con la Formación Frío (localizada en la Cuenca de Burgos; Tabla 2) constituida por intercalaciones de lutita-arenisca. 
Tabla 4. Descripción geológica de las formaciones y unidades litológicas analizadas, número de perfiles sísmicos realizados y valores promedio VS30 por material geológico.

\begin{tabular}{|c|c|c|c|c|c|}
\hline Símbolo & Descripción & $\begin{array}{l}\text { No. de } \\
\text { perfiles }\end{array}$ & $V_{S 30}$ & $\begin{array}{c}\text { Desviación } \\
\text { estándar } \\
\sigma( \pm)\end{array}$ & $\begin{array}{c}\text { Relación } \\
V s / V p\end{array}$ \\
\hline Qs & Suelos & 34 & $* 180$ & 39 & 0.7 \\
\hline Qhoal-L & $\begin{array}{l}\text { Sedimentos cuaternarios lacustres y fluviales, en su mayoría erosionados y distribuidos por ríos y arroyos. La mayor parte de } \\
\text { los poblados están asentados sobre este material y presentan algún grado de cementación en el área de estudio. }\end{array}$ & 411 & 559 & 24 & 0.5 \\
\hline $\mathrm{TCg}$ & $\begin{array}{l}\text { Material aluvial de clastos gruesos, comúnmente cementado por carbonato de calcio, encontrado en regiones como La Petaca } \\
\text { o cubierto por sedimentos aluviales no cementados. }\end{array}$ & 50 & 1310 & 27 & 0.5 \\
\hline $\mathrm{TCz}$ & Calizas lacustres del Pleistoceno aflorantes sobre la carretera Reynosa - Nuevo Padilla. & 2 & 1450 & 70 & 0.6 \\
\hline TmBs & $\begin{array}{l}\text { Flujos basálticos en la poción sur y sureste de la Sierra de San Carlos que cubren rocas sedimentarias cretácicas (Fm. } \\
\text { Méndez). }\end{array}$ & 2 & 1800 & 70 & 0.6 \\
\hline ToGa & $\begin{array}{l}\text { Gabro del Oligoceno constituido por plagioclasa, clinopiroxeno, olivino y óxidos metálicos, expuestos principalmente en } \\
\text { arroyos de la Sierra Chiquita como La Gloria, El Pescado y Rincón Murillo. }\end{array}$ & 2 & 2750 & 70 & 0.6 \\
\hline ToSi & $\begin{array}{l}\text { Rocas sieníticas que afloran en la región central, este y sureste de la Sierra Chiquita y se encuentran emplazadas en unidades } \\
\text { sedimentarias cretácicas; consisten de feldespatos alcalinos y nefelina, clinopiroxenos, anfíboles entre otros. }\end{array}$ & 2 & 2000 & 70 & 0.6 \\
\hline $\mathrm{TpCg}$ & Material aluvial cementado que se ve reflejado en el incremento de sus velocidades. & 2 & 1310 & 41 & 0.5 \\
\hline ToLu-Ar & Intercalaciones de lutita y arenisca de la Fm. Frío, presente en la Cuenca de Burgos. & 2 & 720 & 84 & 0.5 \\
\hline ToAr-Lu & $\begin{array}{l}\text { Secuencia sedimentaria compuesta por alternancia de arenisca y lutita y que representa a la Fm. Vicksburg, encontrada en la } \\
\text { Cuenca de Burgos. }\end{array}$ & 4 & 1500 & 81 & 0.6 \\
\hline TemAr-Lm & $\begin{array}{l}\text { La Fm. Viento, presente en la Cueca La Popa, es una unidad compuesta principalmente por arenisca y que puede presentar } \\
\text { limolita de grano fino a medio en estratos horizontales o inclinados. }\end{array}$ & 2 & 1640 & 70 & 0.7 \\
\hline TeiAr-Lmr & $\begin{array}{l}\text { Depositada en ambientes de estuario y planicie costera, la Fm. Adjuntas consiste de limolita roja y estratos lenticulares de } \\
\text { arenisca de grano fino a medio. Está presente en la Cuenca La Popa. }\end{array}$ & 2 & 1570 & 42 & 0.6 \\
\hline TplcArc-Arn-Ar & $\begin{array}{l}\text { Sedimentos arcillo-arenosos, con intercalaciones variables de arenisca, y que muestra características de barras costeras, que } \\
\text { representan a la Fm. Wilcox en la Cuenca de Burgos. }\end{array}$ & 4 & 1560 & 10 & 0.6 \\
\hline TplcArc & $\begin{array}{l}\text { Fm. Midway, cuyos sedimentos arcillosos, depositados en plataforma media y externa, son equivalentes a los sedimentos de la } \\
\text { Fm. Wilcox, presente en la Cuenca de Burgos. }\end{array}$ & 8 & 1710 & 16 & 0.5 \\
\hline TplcAr & $\begin{array}{l}\text { Areniscas basales y de ambientes pelágicos de la Fm. Velasco (en la Cuenca de Burgos), que representan un importante } \\
\text { marcador del límite } \mathrm{K} / \mathrm{T} \text { en el NE de México. }\end{array}$ & 2 & 1200 & 84 & 0.6 \\
\hline KsAr & $\begin{array}{l}\text { Unidad de mayor espesor del Grupo Difunta, consistente de arenisca de grano grueso y conglomerado y que está presente en la } \\
\text { Cuenca La Popa (Fm. Potrerillos). }\end{array}$ & 4 & 1230 & 24 & 0.5 \\
\hline KsAr-Lu & $\begin{array}{l}\text { Consistente de intercalaciones de arenisca y lutita, la Fm. Muerto, presente en la Cuenca La Popa. Representa un sistema } \\
\text { deltaico dominado por olas; contiene granos líticos volcánicos y plagioclasas indicando una fuente volcánica. }\end{array}$ & 4 & 1420 & 16 & 0.6 \\
\hline KsLu-cal & $\begin{array}{l}\text { Secuencia de lutita y marga marrón oscuro a claro de la Fm. Méndez. Se presenta en estratificación delgada a laminar, en } \\
\text { ocasiones se observan arenisca y caliza arcillosa intercaladas con lutita. }\end{array}$ & 35 & 2149 & 9 & 0.6 \\
\hline $\mathrm{KmsCz}-\mathrm{mg}$ & $\begin{array}{l}\text { Caliza margosa de color gris intercalada con lutita y horizontes bentónicos de tonalidad verde y caliza silicificada de la Fm. } \\
\text { San Felipe. }\end{array}$ & 32 & 2381 & 15 & 0.6 \\
\hline $\mathrm{KsCz}-\mathrm{Lu}-\mathrm{ca}$ & Caliza intercalada con lutita carbonosa de color negro de la Fm. Agua Nueva. & 6 & 2152 & 26 & 0.6 \\
\hline $\mathrm{KmCz}$ & Caliza gris oscuro a negro, de estratificación delgada, con lentes y nódulos de pedernal de la Fm. Cuesta del Cura. & 4 & 1770 & 73 & 0.5 \\
\hline $\mathrm{KmCz}$ & Fm. Tamaulipas Superior, que consta de caliza delgada de estratificación irregular con presencia de lentes de pedernal negro. & 4 & 2330 & 16 & 0.5 \\
\hline $\mathrm{KiCz}-\mathrm{Lu}$ & Intercalación de estratificación delgada a laminar de caliza arcillosa y lutita, rica en amonites, de la Fm. La Peña. & 4 & 1630 & 40 & 0.7 \\
\hline $\mathrm{KiCz}$ & Caliza gruesa de color gris de la Fm. Cupido. Presenta cambios de facies arrecifales de talud de plataforma y de cuenca. & 12 & 3440 & 94 & 0.7 \\
\hline $\mathrm{KiCz}-\mathrm{Lu}$ & $\begin{array}{l}\text { Secuencia de caliza arcillosa color gris oscuro a negro de estratificación delgada y poco competente a la erosión de la Fm. } \\
\text { Taraises. }\end{array}$ & 12 & 2100 & 46 & 0.6 \\
\hline JsKi Lu-Cz-Ar & Secuencia compuesta por intercalaciones de lutita negra fosilífera, arenisca y caliza de la Fm. La Casita. & 11 & 1513 & 46 & 0.5 \\
\hline $\mathrm{JsCz}$ & Fm. Zuloaga, la cual presenta caliza de estratificación delgada a media con presencia de ooides. & 21 & 1760 & 37 & 0.5 \\
\hline JmsEv & $\begin{array}{l}\text { Secuencia de evaporitas (anhidrita, yeso, halita), con aisladas intercalaciones de lutita y arenisca, de la Fm. Minas Viejas, en el } \\
\text { poblado San Pablo de Tranquitas al sur de Galeana, N. L. }\end{array}$ & 6 & 1510 & 18 & 0.6 \\
\hline TRJLR & $\begin{array}{l}\text { Lutita, arenisca bien consolidada y conglomerado, regularmente cementados con tonalidades verde claro, gris verdoso y rojo } \\
\text { violáceo de la Fm. Huizachal al sur de Galeana, N. L. }\end{array}$ & 4 & 1540 & 24 & 0.5 \\
\hline TRJLv-Ri-P-Ar & $\begin{array}{l}\text { Miembro volcánico de la Fm. Huizachal caracterizada por alternancia de lavas, cuerpos riolíticos, piroclásticos y arenisca, } \\
\text { afloramiento encontrado en el poblado del Huizachal al SE de Cd. Vitoria, Tamaulipas. }\end{array}$ & 2 & 1790 & 14 & 0.5 \\
\hline PzpAr-Lu & $\begin{array}{l}\text { Fm. Guacamaya, constituida por arenisca interestratificada con limolita y lutita, presentes en el Cañón La Peregrina, } \\
\text { Tamaulipas. }\end{array}$ & 2 & 2660 & 28 & 0.6 \\
\hline PzcAr-Lu & $\begin{array}{l}\text { Conjunto constituido por arenisca cuarcífera de color gris oscuro con bandas conglomeráticas y lutita negra, que representan a } \\
\text { la Fm. Vicente Guerrero, presente en el Cañón La Peregrina. }\end{array}$ & 4 & 2560 & 24 & 0.6 \\
\hline PzsCz-Lu-Ar & $\begin{array}{l}\text { Fm. Cañón de Caballeros, constituida por secuencias calcáreo-clásticas con conglomerado y arenisca, con gránulos volcánicos. } \\
\text { Se localiza en el Cañón La Peregrina, al oeste de Ciudad Vitoria, Tamaulipas. }\end{array}$ & 2 & 1840 & 70 & 0.6 \\
\hline PzSerp & $\begin{array}{l}\text { Serpentinitas, que representan el segundo miembro del Esquisto Granjeno y que se forman por metamorfismo de bajo grado } \\
\text { de basalto o gabro. }\end{array}$ & 2 & 3110 & 14 & 0.6 \\
\hline PzE & $\begin{array}{l}\text { Presente en el Anticlinorio Huizachal-Peregrina, el Esquisto Granjeno se forma a partir del metamorfismo de bajo grado de } \\
\text { sedimentos parcialmente ricos en arcillas, depositados en una cuenca profunda y cercanos a una fuente volcánica. }\end{array}$ & 2 & 2080 & 98 & 0.6 \\
\hline PrGn & $\begin{array}{l}\text { Encontrado en el Cañón del Novillo a suroeste de Ciudad Victoria, Tamaulipas. El Gneis Novillo representa el basamento } \\
\text { cristalino metamorfizado y de protolito ígneo. }\end{array}$ & 6 & 2105 & 3 & 0.6 \\
\hline
\end{tabular}

$\mathrm{V}_{\mathrm{S} 30}$ : Velocidad de ondas $S$ en los primeros $30 \mathrm{~m}$ del subsuelo; Vp: Velocidad de ondas P; Vs: Velocidad de ondas $\mathrm{S}$; * Velocidad promedio de la capa de suelo con espesores menores a 3 m; Qs: suelos del Cuaternario; Qhoal-L: Aluviones Cuaternarios; TCg: Conglomerado del Terciario; TCz: Calizas Terciarias; TmBs: Basaltos del Terciario medio; ToGa: Gabros del Oligoceno; ToSi: Sienitas del Oligoceno; TpCg: Conglomerado del Plioceno; ToLu-Ar: Lutitas-Areniscas del Oligoceno; ToAr-Lu: Areniscas-Lutitas del Oligoceno; TemAr-Lm: Areniscas-Limolitas del Eoceno medio; TeiAr-Lmr: AreniscasLimolitas rojas del Eoceno inferior; TplcArc-Arn-Ar: Arcillas arenosas-arenisca del Paleoceno; TplcArc: Arcillas del Paleoceno; TplcAr: Areniscas del Paleoceno; KsAr: Areniscas Cretácicas; KsAr-Lu: Areniscas-Lutitas Cretácicas; KsLu-cal: Lutitas calcáreas Cretácicas; KmsCz-mg: Calizas margosas Cretácicas; KsCz-Lu-ca: Caliza-Lutita carbonosa Cretácica; KmCz: Calizas del Cretácico medio; KiCz-Lu: Calizas-Lutitas del Cretácico inferior; KiCz: Calizas del Cretácico inferior; JsKi Lu-Cz-Ar: Lutita-Caliza-Arenisca Jurásico-Cretácicas; JsCz: Calizas Jurásicas; JmsEv: Evaporitas Jurásicas; TRJLR: Lechos rojos del Triásico-Jurásico; TRJLv-Ri-P-Ar: Lavas-Riolitas-Piroclastos-Areniscas: Triásico-Jurásicas; PzpAr-Lu: Areniscas-Lutitas del Pérmico; PzcAr-Lu: Areniscas-Lutitas Carboníferas; PzsCz-Lu-Ar: Calizas-Lutitas-Areniscas Silúricas; PzSerp: Serpentinitas del Paleozoico; PzE: Esquistos Paleozoicos; PrGn: Gneis Precámbrico. 
Esta formación presenta valores de $\mathrm{V}_{\mathrm{S} 30}=720 \mathrm{~m} / \mathrm{s}$, siendo clasificada como sitio clase $\mathrm{C}$.

Los perfiles sísmicos realizados en rocas del Terciario, se llevaron a cabo en tres sub-provincias con características geológicas diferentes en su formación: Cuenca La Popa, Cuenca de Burgos y el Complejo Intrusivo de la Sierra de San Carlos. Las características de estas estructuras geológicas han condicionado las relaciones de velocidades de ondas sísmicas observadas. Los promedios de velocidad $\mathrm{V}_{\mathrm{S} 30}$ medidos en el Complejo Intrusivo de la Sierra de San Carlos fueron los más altos $\left(1800<\mathrm{V}_{\mathrm{S} 30}<2750\right.$; Tablas 3 y 4), clasificándose como sitios clase A (basaltos, gabros y sienitas). Los valores intermedios medidos se ubicaron en la Cuenca La Popa donde variaron entre $1230 \leq \mathrm{V}_{\mathrm{S} 30} \leq 1640$ $\mathrm{m} / \mathrm{s}$. Los perfiles sísmicos realizados se llevaron a cabo en las formaciones siliciclásticas Muerto, Difunta, Potrerillos, Adjuntas y Viento. Las velocidades más bajas corresponden a la Cuenca de Burgos, donde los valores varían entre $720 \leq \mathrm{V}_{\mathrm{S} 30} \leq 1710 \mathrm{~m} / \mathrm{s}$, las cuales se correlacionan con las formaciones Velasco, Midway, Wilcox, Vicksburg y Frío (Tablas 3 y 4 ).

\subsection{Cuaternario}

De manera general, las unidades geológicas del Cuaternario presentan variaciones significativas en las velocidades de propagación de ondas sísmicas. Estas son el resultado del depósito en ambientes geológicos similares a los presentes en la actualidad. Por esta razón, en algunas zonas urbanas distribuidas en todo el mundo, se han realizado cartografías donde se muestra con cierto nivel de detalle estudios de la distribución de las unidades geológicas del Cuaternario, clasificadas de acuerdo a su ambiente de depósito. En algunos casos existe, además, un control en la descripción del tipo de grano, densidad, porosidad y grado de cementación (Wills et al., 2000). Estos factores son los que controlan las velocidades de propagación de las ondas sísmicas y han permitido para algunas zonas urbanas hacer zonificaciones muy detalladas de la distribución de $\mathrm{V}_{\mathrm{S} 30}$. Para la región de estudio, sólo en Monterrey y su área metropolitana se cuenta con ese nivel de detalle (AlvaNiño, 1995; Montalvo-Arrieta et al., 2008). Para el resto del estado, los materiales recientes (Cuaternario) han sido clasificados solamente como material aluvial.

Los principales centros urbanos del área de estudio: Monterrey y su área metropolitana, así como el resto de las principales ciudades del estado (Linares, Cadereyta, Allende y Montemorelos) se encuentran asentadas sobre sedimentos del Cuaternario. De acuerdo a las condiciones climáticas, así como a la disponibilidad de agua, estos centros urbanos se han asentado en la porción oriental de la SMO, así como dentro de la PCG, ocupando desde las laderas de la gran cadena montañosa hasta extensos valles. Estos valles están rellenos por sedimentos erosionados de la SMO, principalmente, conglomerados y sedimentos aluviales. Es común que, parte de la urbanización en la planicie se sitúe sobre terrazas fluviales (Ruiz-Martínez y Werner, 1997). De manera general, se ha documentado que los espesores máximos de los depósitos aluviales en las áreas urbanas de Monterrey y Linares son menores a 25 m (Alva-Niño, 1995; Galván Mancilla, 1996; Prado Gómez, 1997; Ruiz-Martínez y Werner, 1997, Montalvo-Arrieta et al., 2005, 2008). A partir de los trabajos de Montalvo-Arrieta et al. $(2005,2008)$ y los realizados en este estudio, se ha documentado que el tipo de sedimentos aluviales presentes en el área de estudio (aluvión, Qhoal-L) presentan valores promedio de $\mathrm{V}_{\mathrm{S} 30}=$ $559 \mathrm{~m} / \mathrm{s}$ y corresponden con sitios clase C.

\subsection{Determinación de $\mathrm{V}_{\mathrm{S} 30}$}

El cálculo de los valores de $\mathrm{V}_{\mathrm{S} 30}$ en los sitios analizados se obtuvo a partir de la Ecuación 1. Para algunos sitios, ubicados fuera de las zonas urbanas, los valores de $\mathrm{V}_{\mathrm{S} 30}$ fueron asignados de manera directa a partir del valor promedio medido de $\mathrm{V}_{\mathrm{S}}$, debido a que los perfiles de refracción fueron realizados directamente sobre el afloramiento de la formación geológica de interés. De manera particular, para aquellas formaciones con edades del Paleozoico y Mesozoico donde por su revisión en campo se identifican como rocas consolidadas, debido a su grado de compactación, cementación y dureza, se clasificaron como sitios clase A y B. Como se ha descrito, las formaciones que conforman la columna geológica tipo para el área de estudio presentan como mínimo espesores de $50 \mathrm{~m}$, por lo que el valor obtenido de $V_{S}$ puede representar claramente a $V_{S 30}$. Para las formaciones analizadas, se encontró que existe una relación entre el incremento del espesor con el aumento de la velocidad de propagación de ondas sísmicas, como resultado del grado de compactación de los materiales geológicos. Sin embargo, se determinó que las formaciones más arcillosas presentan mayores variaciones en la velocidad de propagación para diferentes profundidades con respecto a formaciones con la ausencia de estos materiales. Un ejemplo característico es la Formación Méndez, la cual tiende a meteorizarse fácilmente por su composición litológica (lutitas, margas). Análisis detallados (Picciotto-Fernández, 2000; Montalvo-Arrieta et al., 2008; Infante et al., 2010) en el área de estudio muestran estas variaciones. PicciottoFernández (2000), empleando una fuente tipo "sparker" en tres pozos perforados en la Facultad de Ciencias de la Tierra de la UANL en Linares, Nuevo León, determinó la estructura de velocidades para esta formación, demostrando además que existe una variación en la velocidad de propagación de ondas $P$ de $3200 \mathrm{~m} / \mathrm{s}$ ( $10 \mathrm{~m}$ de profundidad) a $5400 \mathrm{~m} / \mathrm{s}$ para profundidades mayores a $45 \mathrm{~m}$. Para el mismo sitio, pero empleando perfiles sísmicos de refracción en superficie, Infante et al. (2010) determinaron que para $\mathrm{V}_{\mathrm{p}}$ existen variaciones de $2000 \mathrm{~m} / \mathrm{s}$ cerca de la superficie (4 $\mathrm{m}$ ) hasta más $3000 \mathrm{~m} / \mathrm{s}$ para profundidades mayores a $10 \mathrm{~m}$. En el área metropolitana de Monterrey, Montalvo-Arrieta et al. (2008) observaron que las variaciones de $\mathrm{V}_{\mathrm{P}} \mathrm{y} \mathrm{V}_{\mathrm{S}}$ son de 2140 a $3750 \mathrm{~m} / \mathrm{s}$ y 1200 a $2500 \mathrm{~m} / \mathrm{s}$ respectivamente, 
para los primeros $30 \mathrm{~m}$ del subsuelo. Estas variaciones fueron comúnmente encontradas en aquellas formaciones con altos contenidos de lutitas y/o margas, por ejemplo: La Casita, San Felipe del Mesozoico, o Frío, Vicksburg y Velasco para el Terciario.

Para el caso de formaciones compuestas por calizas (i.e. Cupido/Tamaulipas Inferior, Aurora/Tamaulipas Superior, Cuesta del Cura, entre otras), se encontró que los valores de $\mathrm{V}_{\mathrm{S}}$ no variaron mucho con respecto a la profundidad. Estas formaciones son más resistentes a los procesos de meteorización/erosión, lo cual se vio reflejado con variaciones no significativas de $\mathrm{V}_{\mathrm{s}}$. En la literatura (i.e. Wills et al., 2000) se ha determinado que no se puede asignar valores de $\mathrm{V}_{\mathrm{S}}$ a $\mathrm{V}_{\mathrm{S} 30}$ cuando existen variaciones importantes en las condiciones geológicas en pocos metros de espesor. Casos clásicos se han reportado en la Ciudad de México o en la bahía de San Francisco, donde se ha encontrado que existen variaciones significativas de los valores de $V_{S}$ en decenas de metros. Por ejemplo, para el sitio Alameda Central en la Ciudad de México, se han calculado variaciones importantes en menos de $30 \mathrm{~m}$ de espesor, donde se tienen valores de $\mathrm{V}_{\mathrm{S}}=80 \mathrm{~m} / \mathrm{s}$ para los primeros 7 $\mathrm{m}$ del subsuelo, una inversión de velocidades entre 7 a 30 $\mathrm{m}$ de profundidad con valores de $\mathrm{V}_{\mathrm{S}}$ de $65 \mathrm{~m} / \mathrm{s}$ y a partir de $31 \mathrm{~m}$ de profundidad la $\mathrm{V}_{\mathrm{s}}$ se incrementa a $180 \mathrm{~m} / \mathrm{s}$. Por otro lado, en regiones áridas o semiáridas se ha observado que los sedimentos Cuaternarios no presentan cambios tan significativos en pocas decenas de metros, principalmente cuando los sedimentos aluviales muestran procesos de cementación por medio de caliche (Montalvo-Arrieta et al., 2005, 2008; Scott et al., 2006). Por el contrario, la presencia de este cementante genera que exista un incremento en la velocidad de propagación de ondas sísmicas de los depósitos aluviales (en los primeros metros del subsuelo), como lo han observado Scott et al. (2006) y Montalvo-Arrieta et al. $(2005,2008)$ para ciudades como Las Vegas en EE.UU., y Linares y Monterrey, respectivamente. Estas observaciones motivan a que se pueda utilizar el valor $\mathrm{V}_{\mathrm{S}} \operatorname{como} \mathrm{V}_{\mathrm{S} 30}$, principalmente para formaciones consolidadas del Terciario, Mesozoico, Paleozoico y Precámbrico. Para el caso de las zonas urbanas, los valores de $\mathrm{V}_{\mathrm{S} 30}$ fueron obtenidos por la Ecuación 1. Como se mencionó anteriormente, para los municipios de Linares y Monterrey, se pudieron realizar correlaciones de las estructuras de velocidades obtenidas con información de pozos. Esto permitió verificar los datos obtenidos a partir de los perfiles sísmicos con clasificaciones geotécnicas (Rodríguez-Marek et al., 2001) para la determinación de efectos sísmicos de sitio. Esto ha sido documentado por Montalvo-Arrieta et al. (2005, 2008). Con los valores obtenidos de $\mathrm{V}_{\mathrm{S} 30}$ para cada una de las formaciones y unidades litológicas analizadas descritas en las Tablas 3 y 4 y en Montalvo-Arrieta et al. (2005, 2008), se procedió a generar el mapa de la distribución de $\mathrm{V}_{\mathrm{S} 30}$ para todo el estado.

\section{Generación del mapa de la distribución de $V_{S 30}$ para el estado de Nuevo León}

Una vez definido el detalle del mapa geológico a utilizar (escala 1:250000), se procedió a la asignación de los valores de $\mathrm{V}_{\mathrm{S} 30}$, descritos en la Tabla 3, para cada una de las unidades geológicas presentes en el área de estudio. Esto se llevó a cabo a través de un sistema de información geográfica en donde se implementó la información geológica mostrada en las Figuras 2 y 3. La Figura 4 muestra el mapa de la distribución de la respuesta sísmica de sitio a partir de velocidades de ondas de corte. Como se puede observar en el mapa, los sitios clase $A\left(V_{\mathrm{S} 30}>1500 \mathrm{~m} / \mathrm{s}\right)$ se localizan principalmente en la SMO y corresponden principalmente con rocas consolidadas del Mesozoico. Los sitios Clase $\mathrm{B}\left(760<\mathrm{V}_{\mathrm{S} 30}<1500 \mathrm{~m} / \mathrm{s}\right)$ se ubican en sedimentos de edad Terciaria, los cuales corresponden a formaciones que se encuentran en proceso de consolidación, así como

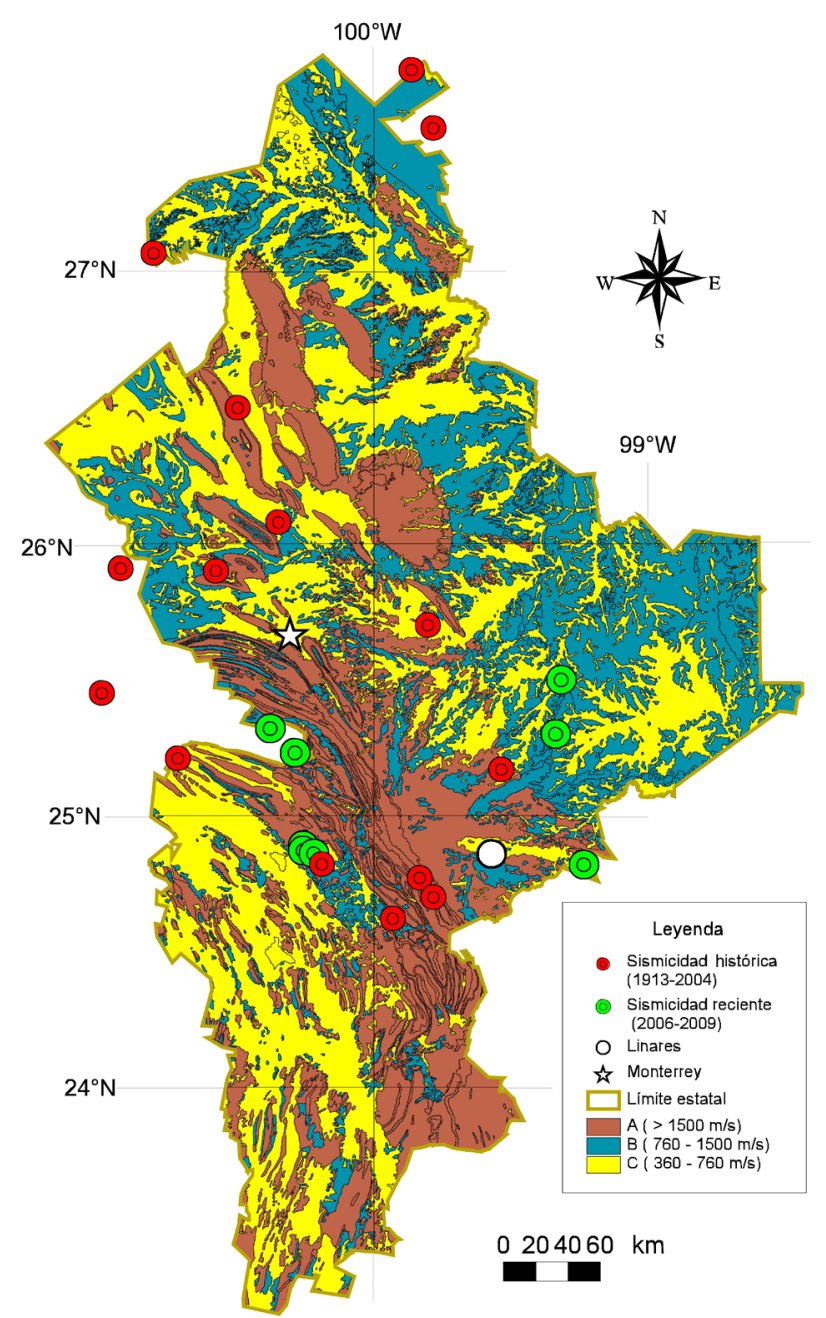

Figura 4. Mapa de la distribución de $\mathrm{V}_{\mathrm{S} 30}$ para el Estado de Nuevo León, a partir de perfiles de refracción sísmica e información geológica. La clasificación A, B y C es de acuerdo a las especificaciones de la NEHRP. Los círculos rojos indican la sismicidad histórica (1913-2004) y los círculos verdes señalan la sismicidad reciente (2006-2009), registradas en el área. 
conglomerados fuertemente cementados. Finalmente, los sitios Clase $\mathrm{C}\left(360<\mathrm{V}_{\mathrm{S} 30}<760 \mathrm{~m} / \mathrm{s}\right)$ corresponden con depósito aluviales del Cuaternario, cuyo valor promedio de $\mathrm{V}_{\mathrm{S} 30}$ fue de $560 \mathrm{~m} / \mathrm{s}$. Algunos sitios, con valores más altos que este promedio, se relacionan con la presencia de cementante (caliche), el cual genera que el material se encuentre parcialmente consolidado. Las áreas urbanas de Monterrey y Linares se ubican sobre sitios clase C.

\section{Discusión y conclusiones}

En este trabajo se ha generado el primer mapa de las condiciones sísmicas de sitio, escala 1:250000, para el estado de Nuevo León. Este se preparó a partir de la integración de información geológica (formaciones geológicas y/o unidades litológicas) con propiedades físicas, tal como el promedio de velocidades de $\mathrm{V}_{\mathrm{S} 30}$, determinado para cada una de estas formaciones y/o unidades litológicas. Los resultados obtenidos en este estudio serán de utilidad para generar mapas de riesgo sísmico a nivel regional, en donde las velocidades de propagación de ondas de corte pueden ser utilizadas para determinar el efecto sísmico de sitio. Esta información también puede integrarse a estudios de efectos de contrastes de impedancias entre materiales someros $(\leq 30 \mathrm{~m})$ y sedimentos profundos $(>30 \mathrm{~m})$ y en el análisis geométrico de unidades litológicas.

Para la generación del mapa de las condiciones sísmicas de sitio, se utilizó la clasificación de suelos propuesta por la NEHRP, la cual se ha incorporado a diferentes códigos de construcción de diversos países. La correlación entre geología y velocidades de propagación de ondas $\mathrm{S}$ y/o $\mathrm{V}_{\mathrm{S} 30}$ se llevó a cabo agrupando las unidades litológicas con edades y propiedades similares, utilizando el concepto de formación geológica. Se realizaron 708 perfiles sísmicos distribuidos en localidades de los estados de Nuevo León, Coahuila y Tamaulipas, los cuales cubrieron la columna litológica tipo para el área de estudio. Los perfiles realizados incluyeron variaciones en ambientes de sedimentación de formaciones geológicas, las cuales se distribuyen en diferentes localidades del estado. Esto permitió dar cobertura a los diferentes tipos de litologías presentes en la región. De manera particular, fue posible establecer las variaciones en espesores de material aluvial presente en las zonas urbanas de Linares y Monterrey. A partir de la clasificación de la NERHP, se determinó que las rocas precámbricas, paleozoicas y mesozoicas se clasifican como sitios clase $\mathrm{A}\left(\mathrm{V}_{\mathrm{S} 30} \geq 1500 \mathrm{~m} / \mathrm{s}\right)$. Las rocas de edad terciaria se clasifican como materiales tipo $\mathrm{B}$, ubicándose en el intervalo $760 \leq \mathrm{V}_{\mathrm{S} 30} \leq 1500 \mathrm{~m} / \mathrm{s}$. Los sedimentos del Cuaternario presentaron valores de $\mathrm{V}_{\mathrm{S} 30}$ entre 360 a 760 $\mathrm{m} / \mathrm{s}$, clasificándose como sitios clase $\mathrm{C}$.

La mayor parte de los perfiles sísmicos en sedimentos del Cuaternario se realizaron en las zonas urbanas de Monterrey y Linares. En estas áreas, además, se contó con información de pozos, lo cual permitió correlacionar los valores de obtenidos de $\mathrm{V}_{\mathrm{S} 30}$ con otro tipo de clasificaciones geotécnicas, como la propuesta por Rodríguez-Marek et al. (2001). Para estas zonas, de acuerdo con lo observado por Montalvo-Arrieta et al. $(2005,2008)$, se determinó que la variación de la respuesta de sitio definida a través de $\mathrm{V}_{\mathrm{S} 30}$ está condicionada por dos factores: la presencia de paleocanales y el grado de cementación por caliche de los materiales aluviales, esto último particularmente para regiones caracterizadas por altas tasas de evapotranspiración, como es el caso del noreste de México. A mayor grado de cementación, la velocidad de propagación de $\mathrm{V}_{\mathrm{S} 30}$ alcanzó valores cercanos a los $760 \mathrm{~m} / \mathrm{s}$.

A partir de los resultados obtenidos se encontró que, las unidades litológicas en el área estudiada, presentaron una correlación en términos de las condiciones sísmicas de sitio y la edad de estas, como lo definieron Park y Elrick (1998). Las unidades del Cuaternario asociadas con aluvión presentan las velocidades más bajas (sitios Clase C), el Terciario queda expresado como rocas suaves (sitios clase B) y el Mesozoico y Paleozoico corresponden a rocas duras o de basamento (sitios Clase A).

De manera general, la determinación de los valores de velocidades de propagación de ondas de corte para sedimentos someros son necesarios para la evaluación de la capacidad de carga de los materiales, así como para estudios de la evaluación de riesgo sísmico en el diseño de estructuras civiles grandes y complejas. Los resultados aquí reportados no son suficientemente precisos para este tipo de trabajo, donde se necesitan estudios más específicos y altamente detallados. La correlación entre formaciones/ unidades geológicas y las velocidades de propagación de ondas sísmicas deberán ser consideradas en los límites entre formaciones, principalmente en los contactos Mesozoico-Cuaternario, Terciario-Cuaternario, donde los cambios en los valores de $\mathrm{V}_{\mathrm{S} 30}$ pueden ser significativos. Debido a la escala de los mapas geológicos, pueden existir errores en la cartografía de la unidad litológica, por lo que, el límite entre formaciones puede variar en decenas de metros. Para estos casos son necesarios estudios de detalle, tanto geológico como sísmico, para determinar estas variaciones, principalmente en zonas urbanas donde se planee la construcción de obras civiles. En la medida en que a esta base de datos se le incorpore más información geotécnica, el mapa regional tenderá a actualizarse con un análisis más detallado de la respuesta sísmica de sitio. Por otro lado, también hay que considerar que este mapa no incluye efectos relacionados con la geometría de cuencas y otros efectos relacionados con la estructura somera. Este mapa provee una primera aproximación de la respuesta sísmica de los materiales someros, cuya utilidad será de importancia en la planeación del uso de suelo, desarrollo urbano, así como para el cálculo del riesgo sísmico u otros peligros geológicos. Particularmente, el mapa será de utilidad considerando la evidencia de actividad sísmica y la escasa instrumentación sísmica disponible en la región. 


\section{Agradecimientos}

Este trabajo ha sido parcialmente apoyado por los proyectos: Apoyo a la Incorporación de Nuevos PTC clave: PROMEP/103.5/03/2552, PAICYT-UANL CT877-04 y CONACYT 25637. El segundo autor (LGRZ) agradece al CONACYT por la beca otorgada para el desarrollo de su tesis doctoral en el Posgrado de la Facultad de Ciencias de la Tierra, UANL (Doctorado en Ciencias, Especialidad en Geociencias).

\section{Referencias}

Aguilera, J.G., 1888, Estudio de los fenómenos sísmicos del 3 de mayo de 1887: Anales del Ministerio de Fomento de la República Mexicana $10,5-56$.

Alva-Niño, E., 1995, Datos base y metodología para la elaboración de una carta ingeniero-geológica de la zona metropolitana de Monterrey, N. L., México: Linares, Nuevo León, México, Universidad Autónoma de Nuevo León, Tesis de Licenciatura, 82 p.

Boore, D.M., Joyner, W.B., Fumal, T.E., 1994, Estimation of response spectra and peak accelerations from western North American earthquakes: an interim report, Part 2: U.S. Geological Survey Open-File Report 94-127, 40 p.

Borcherdt, R.D., 1994, Estimates of site-dependent response spectra for design (methodology and justification): Earthquake Spectra, 10, $617-653$.

Bryant, W. R., Lugo, J., Córdova, C., Salvador, A., 1991, Physiography and bathymetry, in A. Salvador (ed.), The Gulf of Mexico Basin: Boulder, Colorado, Geological Society of America, 13-30.

Buffler, R.T., Sawyer, D.S., 1985, Distribution of crust and early history, Gulf of Mexico basin: Gulf Coast Association of Geological Societies Transactions, 35, 333-334.

Building Seismic Safety Council (BSSC), 1994, NEHRP recommended provisions for seismic regulations for new buildings Part 1 Provisions, FEMA 222A: Washington, D.C., Federal Emergency Management Agency, $290 \mathrm{p}$.

Campa, M.F., Coney, P.J., 1983, Tectono-stratigraphic terrains and mineral resource distribution in Mexico: Canadian Journal of Earth Sciences, 20, 1040-1051

Carrillo-Bravo, J., 1961, Geología del anticlinorio Huizachal Peregrina al N-W de Ciudad Victoria: Boletín de la Asociación Mexicana de Geólogos Petroleros, XII, 1-98.

Castillo-Rodríguez., H., 1988, Zur Geologie des kristallinen Grundgebirges der Sierra Madre Oriental - insbesondere des Granjeno- SchieferKomplexesim Südteil des Huizachal-Peregrina-Antiklinoriums (Raum Ciudad Victoria, Tamaulipas, Mexiko): Münster, Renania del Norte-Westfalia, Alemania, Universidad de Münster, Tesis de Maestría, $138 \mathrm{p}$.

Coney, P.J., 1978, Mesozoic-Cenozoic Cordilleran plate tectonics, in Smith. R.B., Eaton, G.P. (eds.), Cenozoic tectonics and regional geophysics of the western Cordillera: Geological Society of America Memoir, 152, 33-50.

Cossío-Torres, T., 1988, Zur Geologie der Sierra Madre Orientalinsbensondere des Novillo-Gneis-Komplexes- im Südteil des Huizachal-Peregrina-Antiklinoriums (Raum Ciudad Victoria, Tamaulipas, Mexiko): Münster, Renania del Norte-Westfalia, Alemania, Universidad de Münster, Tesis de Maestría, 99 p.

Crone, A.J., De Martini, P.M., Machette, M.N., Okumura, K., Prescott, J., 2003, Paleoseismicity of two historically quiescent faults in Australia: implications for fault behavior in stable continental regions: Bulletin of the Seismological Society of America, 93, 1913-1934.

Damon. P.E., Shafiqullah, M., 1991, Chronology of Mesozoic magmatism in Sonora, northwestern Mexico: Geological Society of America Abstracts with Programs, 25, 127.

Denison, R.E., Burke, W.H., Jr., Hetherington, E.A., Otto, J.B., 1970, Basement rock framework of parts of Texas southern New Mexico and northern Mexico, in Seeward, K.O., Sundeen, D.A. (eds.), The Geologic framework of the Chihuahua Tectonic Belt: Midland, Texas, West Texas Geological Society, 3-14.

Dickinson, W.R., 1981, Plate tectonic evolution of the southern Cordillera, in Dickinson, W.R. and Payne, W.D., eds., Relations of tectonics to ore deposits the southern Cordillera: Arizona Geological Society Digest, 14, 481-509.

Doser, D.I., 1987, The 16 August 1931 Valentine, Texas, earthquake: evidence for normal faulting in west Texas: Bulletin of the Seismological Society of America, 77, 2005-2017.

Doser, D.I., Rodriguez, J., 1993, The seismicity of Chihuahua, Mexico, and the 1928 Parral earthquake: Physics of the Earth and Planetary Interiors, 78, 97-104.

Echánove, O., 1986, Geología Petrolera de la Cuenca de Burgos, Consideraciones Geológico-Petroleras: Boletín de la Asociación Mexicana de Geólogos Petroleros, XXXVIII, 3-74.

Eguiluz de Antuñano, S., Aranda-García, M., Marrett, R., 2000, Tectónica de la Sierra Madre Oriental, México: Boletín de la Sociedad Geológica Mexicana, 53, 1-26.

Flawn, P.T., Díaz-González., T., 1959, Problems of Paleozoic tectonics in north-central and northeastern Mexico: American Association of Petroleum Geologists Bulletin, 43, 224-230.

Fries, C. Jr., Rincón-Orta, C., Silver, L.T., McDowell, F.W., SolorioMunguía, J., Schmitter-Villada, E., Cserna, Z., 1974 (1975), Nuevas aportaciones a la geocronología de la Faja Tectónica Oaxaqueña: Boletín de la Asociación Mexicana de Geólogos Petroleros, 26, 157-182.

Fries, C. Jr., Schmitter-Villada, E., Damon, P.E., Livingston, D.E., Erickson, R., 1962, Edad de las rocas metamórficas en 1os Cañones de La Peregrina y de Caballeros, parte centro-occidental de Tamaulipas: Revista del Instituto de Geología UNAM, 64, 55-69.

Frohlich, C., Davis, S.D., 2002, Texas earthquakes: University of Texas Press, Austin, Texas, 275 p.

Galván-Mancilla, S.M., 1996, Cartografía hidrogeológica de la terraza baja entre Hualahuises y Linares, N. L: Nuevo León, México, Universidad Autónoma de Nuevo León, Facultad de Ciencias de la Tierra, Tesis de Licenciatura, $63 \mathrm{p}$.

Galván-Ramírez, I.N., Montalvo-Arrieta, J.C., 2008, The historical seismicity and prediction of ground motion in northeast Mexico: Journal of South American Earth Sciences, 25, 37-48.

García-Acosta, V., Suárez-Reynoso, G., 1996, Los sismos en la historia de México: México, Universidad Nacional Autónoma de México, 718 p.

Garrison, J.R., Ramírez- Ramírez, C., Long, L.E., 1980, Rb-Sr isotropic study of the ages and provenance of Precambrian granulite and Paleozoic greenschist near Ciudad Victoria, Mexico, in Plier, R.J., (ed.), The origin of the Gulf of Mexico and the early opening of the central North Atlantic Ocean: Baton Rouge, Louisiana, E.UA., Louisiana State University, 37-49 p.

Goldhammer, R.K., 1999, Mesozoic sequence and paleogeographic evolution of northeast Mexico: Geological Society of America Special Paper, 340, 53 p.

Götte, M., 1990, Halotektonishe Deformationsprozesse in Sulfatgesteinen der Minas Viejas-Formation (Oben-Jura) in der Sierra Madre Oriental, Nortdost-Mexiko: Darmstadt, Hesse, Alemania, Universidad Técnica de Darmstadt, Tesis Doctoral, $270 \mathrm{p}$.

Grajales-Nishimura, J.M., Terrell, D.J., Damon, P.E., 1992, Evidencias de la prolongación del arco magmático cordillerano del Triásico Tardío - Jurásico en Chihuahua, Durango y Coahuila: Boletín de la Asociación Mexicana de Geólogos Petroleros, 42, 1-18.

Gursky, H.J., 1996, Paleozoic Stratigraphy of the Peregrina Canyon area, Sierra Madre Oriental, NE México: Zentralblatt für Geologie und Palaontologie, 7/8, 973-989.

Hanumantharo, C., Ramana, G.V., 2008, Dynamic soil properties for microzonation of Delhi, India: Journal of Earth System Science, 117, 719-730. 
Holzer, T.L., Padovani, A.C., Bennett, M.J., Noce, T.E., Tinsley III, J.C., 2005, Mapping NEHRP $\mathrm{V}_{\mathrm{S} 30}$ site classes: Earthquake Spectra, 21, 161-177.

Infante, V., Gallardo, L.A., Montalvo-Arrieta, J.C., Navarro de León, I., 2010, Lithological classification assisted by the joint inversion of electrical and seismic data at a control site in northeast Mexico: Journal of Applied Geophysics, 70, 93-102.

Instituto Nacional de Estadística, Geografía e Informática (INEGI), 2006, Datos vectoriales digitales escala 1:250000: Aguascalientes, Aguascalientes, México, Instituto Nacional de Estadística Geografía e Informática, CD-ROM.

King, P.B., 1942, Permian of west Texas and southeastern New Mexico: American Association of Petroleum Geologists Bulletin, 26, 535-763.

López-Ramos, E., 1983, Geología general y de México tomo 2: México, D.F., Universidad Nacional Autónoma de México - Instituto de Geología- Editorial Escolar, 453 p.

Michalzik, D., 1988, Trias Biss tiente Unter-Kreide der nordöstlichen Sierra Madre Oriental, Mexico, Fazielle Entwicklung eines passiven Kontinentalrandes: Darmstadt, Hesse, Alemania, Universidad Técnica de Darmstadt, Tesis Doctoral, 247 p.

Midorikawa, S., Matsuoka, M., Sakugawa, K., 1994, Site effects on strong-motion records observed during the 1987 Chiba-Ken-TohoOki, Japan, earthquake: Proceedings of the 9th Japan Earthquake Engineering Symposium, 3, E085-E090.

Montalvo Arrieta, J.C., 2009, Reporte de sismicidad registrada en el estado de Nuevo León para el periodo 14 al 21 de junio de 2009: Linares, Nuevo León, México, Universidad Autónoma de Nuevo León Protección Civil del Estado de Nuevo León, 14 p.

Montalvo-Arrieta, J.C., Quintanilla, Y., Tamez, A., Meneses, M., Ramos, L., Masuch, D., 2005, Microzonation of Linares region (northeast Mexico), based on geology and shear-wave velocity (VS30): Geofísica Internacional, 44, 331-340.

Montalvo-Arrieta, J.C., Cavazos-Tovar, P., Navarro de León, I., AlvaNiño, E., Medina-Barrera, F. 2008, Mapping Seismic Site Classes in Monterrey Metropolitan Area, northeast Mexico: Boletín de la Sociedad Geológica Mexicana, 60, 147-157.

Muir, J.M., 1936, Geology of the Tampico Region, Mexico: Tulsa, Oklahoma, E.U.A, American Association of Petroleum Geologists, $280 \mathrm{p}$.

Natali, S.G., Sbar, M.L., 1982, Seismicity in the epicentral region of the 1887 northeastern Sonora earthquake, Mexico: Bulletin of the Seismological Society of America, 72, 181-196.

Ortega-Gutiérrez, F., Sedlock, R.R., Speed, R.C., 1994, Phanerozoic tectonic evolution of Mexico, in Speed, R.C. (ed.), Phanerozoic evolution of North America continental-oceans transitions: Boulder, Colorado, Geological Society of America,, Summary volume to accompany the DNAG Continent-Ocean Transect Volume, 265-306.

Ortega-Gutiérrez, F., Ruiz, J., Centeno-García, E., 1995, Oaxaquia, a Proterozoic microcontinent accreted to North America during the late Paleozoic: Geology, 23, 1127-1130.

Padilla y Sánchez, R.J., 1982, Geologic Evolution of the Sierra Madre Oriental between Linares, Concepción del Oro, Saltillo, Monterrey, México: Austin, Texas, E.U.A., Universidad de Texas en Austin, Tesis Doctoral, $217 \mathrm{p}$.

Padilla y Sánchez, R.J., 1985, Las estructuras de la Curvatura de Monterrey, Estados de Coahuila, Nuevo León, Zacatecas y San Luis Potosí: Revista del Instituto de Geología UNAM, 6, 1-20.

Padilla y Sánchez, R.J., 1986, Post-Paleozoic Tectonic of Northeast Mexico and its Role in the evolution of the Gulf of Mexico: Geofísica Internacional, 25, 157-207.

Park, S., Elrick, S., 1998, Predictions of shear-wave velocities in southern California using surface geology: Bulletin of the Seismological Society of America, 88, 677-685.

Petersen, M., Bryant, W., Cramer, C., Reichle, M., Real, C., 1997, Seismic ground-motion hazard mapping incorporating site effects for Los Angeles, Orange, and Ventura counties, California: Bulletin of the
Seismological Society of America, 87, 249-255.

Picciotto-Fernández, A.R., 2000, Análisis de datos de tomografía sísmica en un medio anisótropo: Linares, Nuevo León, México, Universidad Autónoma de Nuevo León, Tesis de Licenciatura, 66 p.

Prado-Gómez, E., 1997, Aplicación de métodos geofísicos (SEV's) para investigar zonas de canales de gravas en el área de HualahuisesLinares, Nuevo León, México: Linares, Nuevo León, México, Universidad Autónoma de Nuevo León, Tesis de Licenciatura, 107 p.

Ramírez-Ramírez, C., 1992, Pre-Mesozoic geology of Huizachal-Peregrina anticlinorium, Ciudad Victoria, Tamaulipas, and adjacent parts of eastern Mexico: Austin, Texas, E.U.A., Universidad de Texas en Austin, Tesis Doctoral, $318 \mathrm{p}$.

Roca, A., Oliveira, C.S., Ansal, A., Figueras, S., 2008, Local site effects and microzonation, in Oliveira, C.S., Roca, A., Goula, X. (eds.), Assessing and Managing Earthquake Risk: Geo-scientific and Engineering Knowledge for Earthquake Risk Mitigation, developments, tools, techniques: Dordrecht, Holanda, Springer, 67-89.

Rodríguez Cabo, J., 1946, Fenómenos geológicos en General Terán, NL: Boletín de la Sociedad Geológica Mexicana, XII, 7-43.

Rodríguez-Marek, A., Bray, J.D., Abrahamson, N.A. 2001, An empirical geotechnical seismic site response procedure: Earthquake Spectra, 17, 65-87.

Ruiz,-Martínez, M.A., Werner, J., 1997, Research into the Quaternary sediments and climatic variations in NE Mexico: Quaternary International, 43/44, 145-151.

Salas-Pizá, G., 1970, Evaluación geológico-minera del distrito asbestífero del Cañón del Novillo, Ciudad Victoria, Tamaulipas: México, D.F., Consejo de Recursos Naturales no Renovables, 71, 20 p.

Salvador, A., 1987, Late Triassic paleogeography and origin of Gulf of Mexico basin: American Association of Petroleum Geologists Bulletin, 71, 419-451.

Scott, J.B., Rasmussen, T., Luke, B., Taylor, W.J., Wagoner, J.L., Smith, S.B., Louie J.N., 2006, Shallow shear velocity and seismic microzonation of the urban Las Vegas, Nevada Basin: Bulletin of the Seismological Society of America, 96, 1068-1077.

Sedlock, R.L., Ortega-Gutiérrez, F., Speed, R.C., 1993, Tectonostratigraphic terranes and tectonic evolution of Mexico: Geological Society of America Special Paper, 278, 146 p.

Stein, S., 2007, Approaches to continental intraplate issues, in Stein, S., Mazzotti, S. (eds.), Continental intraplate earthquakes: science, hazard, and policy issues: Geological Society of America Special Paper, 425, 1-16.

Stewart, J.P., Liu, A.H., Choi, Y., 2003, Amplification factors for spectral acceleration in tectonically active regions: Bulletin of the Seismological Society of America, 93, 332-352.

Tinsley, J.C., Fumal, T.E., 1985, Mapping quaternary sedimentary deposits for areal variations in shaking response, in Ziony, J.E. (ed.), Evaluating earthquake hazards in the Los Angeles region an Earth-Sience perspective: U.S. Geological Survey Professional Paper 1360, 101-126.

Visser, W.A., 1980, Geological Nomenclature: Royal Geological and Mining Society of the Amsterdam, Países Bajos, 540 p.

Williams, R.A., Wood, S., Stephenson, W.J., Odum, J.K., Meremonte, M.E., Street, R., Worley, D., 2003, Surface seismic refraction/reflection measurement determinations of potential site resonances and the areal uniformity of NEHRP site class D in Memphis, Tennessee: Earthquake Spectra, 19, 159-189.

Wills, C.J., Silva, W., 1998, Shear wave velocity characteristics of geologic units in California Earthquake Spectra, 14, 533-556.

Wills, C.J., Petersen, M., Bryant, W.A., Reichle, M., Saucedo, G.J., Tan, S., Taylor, G., Treiman, J., 2000, A site-conditions map for California based on geology and shear-wave velocity: Bulletin of the Seismological Society of America, 90, 187-208.

Wills, C.J., Clan, K.B., 2006, Developing a map of geologically defined site-condition categories for California: Bulletin of the Seismological Society of America, 96, 1483-1501. 
Wilson, J.L., 1990, Basement structural controls on Mesozoic carbonate facies in northeastern Mexico-A review, in Tucker, M.E., Wilson, J.E., Crevello, P.D., Sarg, J.R., Read, J.F. (eds.), Carbonate platforms: facies, sequences, and evolution: International Association of Sedimentologists Special Publication, 9, 235-255.

Woods, R.D., Salvador, A., Miles, A.E., 1991, Pre-Triassic, in Salvador, A. (ed.), The Gulf of Mexico Basin: Boulder, Colorado, Geological Society of America, Geology of North America, 109-129.
Xie, J., 1998. Spectral inversion of Lg from earthquakes: a modified method with applications to the 1995, Western Texas earthquake sequence: Bulletin of the Seismological Society of America, 88, 1525-1537.

Manuscrito recibido: Diciembre 7, 2009.

Manuscrito corregido recibido: Agosto 22, 2010.

Manuscrito aceptado: Septiembre 10, 2010. 